\title{
EULER'S ELASTICA AND BEYOND
}

\author{
SHIGEKI MATSUTANI
}

Communicated by Ivaïlo M. Mladenov

\begin{abstract}
In 1691, James (Jacob) Bernoulli proposed a problem called elastica problem: What shape of elastica, an ideal thin elastic rod on a plane, is allowed? Daniel Bernoulli discovered its energy functional, Euler-Bernoulli energy function, and the minimal principle of the elastica. Using it, Euler essentially solved the problem in 1744 by developing the variational method, elliptic integral theory and so on. This article starts with a review of its mathematical meaning and historical background. After that we present one of its extensions, statistical mechanics of elastica as a model of the DNA and the large polymers. We will call it a quantized elastica, and show that it is connected with the modified Korteweg-de Vries hierarchy, loop space, submanifold Dirac operators, moduli spaces of the real hyperelliptic curves and so on. By reviewing the other extensions of the elastica problem, We will see that elastica is in the center of mathematics even now.
\end{abstract}

\section{Introduction}

In a daily life, we encounter various shapes. Leonardo da Vinci (1452-1519) observed these various shapes as an artist, a researcher of medicine, a scientist and an engineer, and sketched them on his notes. His purpose might be to imitate Creator's works and sometimes to control them by understanding their mechanism though we cannot know his net purpose exactly. A prototype of elastica, the main subject of this article, also appeared in his note [72] where he roughly drew a bent thin elastic beam, by considering what shape is obtained when an elastic beam was bent. The drawing might be due to the motivation from applied science to control bridges and beam in architecture. After then, the elastic beams is a theme in mathematical science like a catenary (a curve of a chain in gravitational force with its fixed endings) and Galileo Galilei (1564-1642) also dealt with them in his famous book "Two New Sciences" published in 1638 [22]. Galileo investigated the breaking of a beam by terminal load and the resistance force from the beam.

Following these studies, James (Jacob or Jacques) Bernoulli (1654-1705) proposed the elastica problem: What shape we obtain when an elastica, an ideal 
infinitesimally thin elastic rod without stretching on a plane, is bent? Roughly speaking, the problem is to find what shape a string of guitar on a plane has. It should be noted that the elastica problem is not directly related to string in the string theory [47]. This problem was essentially solved by Euler at 1744 but similar problems have been still studied by several researchers even nowadays.

In this article, following the references $[71,72,74]$, we will review the studies of Bernoulli's and Euler from a historical point of view and mention an attempt, a quantization of elastica, that we step beyond their studies.

Some of the readers might have a question why we choose the topic from the seventeenth and eighteenth centuries. It is not only due to personal preferences. One of the reasons is that these studies of Bernoulli's and Euler on elastica are prototypes of harmonic map theory, nonlinear integrable theory, differential geometry, algebraic geometry, elliptic function theory, theory of moduli of elliptic curves and so on. For example, the studies of the nonlinear integrable differential equations, such as soliton equations, began in 1960's whereas the modern studies of the extrinsic geometries, which were considered as an extreme points of certain energy functional, began in 1980's. Both progress even now but their origin can be found in the theory of elastica. In other words, studies of Bernoulli's and Euler do not rust even in the twenty-first century.

The second reason is that the studies of elastica are among the most important examples of a collaboration of many fields in mathematics including applied mathematics and mathematical physics. The shape is a concerned object even nowadays. In order to understand the shapes as a subject in applied mathematics, Bernoulli's and Euler started to study them and found the abstract concepts: description of curves, i.e., primitive differential geometry, variational principle and method, harmonic map theory, elliptic integral theory, i.e., beginning of algebraic geometry and elliptic function theory, moduli of elliptic curves, classification of solution space with topological number, and so on. They applied the abstract theories to the elastica problem. Steps from the concrete to the abstract, and from the abstract to the concrete were the style of their study. In order to solve a physical or mathematical problem, if need, they created the mathematical tools and theories, and assembled these tools and mathematics in different fields. Then they solved the problem. We believe that such strategies are crucial even now.

In $\S 2$, we will review these studies on the elastica in detail. In $\S 3$, we will mention an attempt to step beyond the studies of Bernoulli's and Euler, which is related to the shape of DNA. When we consider statistical mechanics of elastica in a heat bath, we encounter a generalization of the elastica problem. 
The present author had studied the problem for two decades [44-55] with coauthors [56, 57]. We have called it the quantized elastica problem, which needs much wider fields of mathematics and physics, i.e., modified Korteweg-de Vries hierarchy, hyperelliptic curves, Kleinian $\sigma$ functions, loop spaces, functional integrals, moduli space of hyperelliptic curves, cohomology of fibrations and so on.

Recently there appear nice reviews of Euler's elastica [17, 26, 41, 66, 67]. One of our purposes is to show the strategies of Euler and Bernoulli's via the elastica problem, which is partially overlapped with these reviews. However we have another purpose which is to provide how the results of Euler's elastica are connected with modern mathematics, via quantized elastica. The connection is regarded as the classical-quantum correspondence. In fact, every relation of Euler's elastica in this article is generalized to one in quantized elastica through the generalization of elliptic curves to hyperelliptic curves. The quantized elastica gives classification of the loop space of the two-sphere $\Omega S^{2}$ in category of regular analytic functions. Elastica problem is still alive even nowdays and we would like to mention that we should inherit the style of founders, at least to the quantized elastica problem.

In this article, $\mathbb{C}, \mathbb{R}$ and $\mathbb{Z}$ denote the sets of complex numbers, real numbers and integers respectively.

\section{Euler's Elastica}

\subsection{Geometry of the Plane Curve}

Before entering into the history of elastica, we will prepare a primary differential geometry. Let $s$ be a parameter of a curve defined on a plane such that $Z(s)=$ $X(s)+\sqrt{-1} Y(s)$. When we measure the length of the arc by the measure of the plane itself, i.e., the induced metric, the length is called arclength. The parameter $s$ is the arclength if and only if it satisfies the condition

$$
\left|\partial_{s} Z\right|=1
$$

where $\partial_{s}:=\mathrm{d} / \mathrm{d} s$. Here $\partial_{s} Z$ is a tangential vector and can be expressed as $\mathbf{t}:=\partial_{s} Z=\mathrm{e}^{\sqrt{-1} \phi}$ using the tangential angle $\phi$ whereas the normal vector is $\mathbf{n}=\sqrt{-1} \mathbf{t}$. The Frenet-Serret relation

$$
\left(\begin{array}{cc}
\partial_{s} & -k \\
k & \partial_{s}
\end{array}\right)\left(\begin{array}{l}
\mathbf{t} \\
\mathbf{n}
\end{array}\right)=0
$$

holds. Here $k:=\partial_{s} \phi$ is the curvature (the inverse of curvature radius) of the curve. 


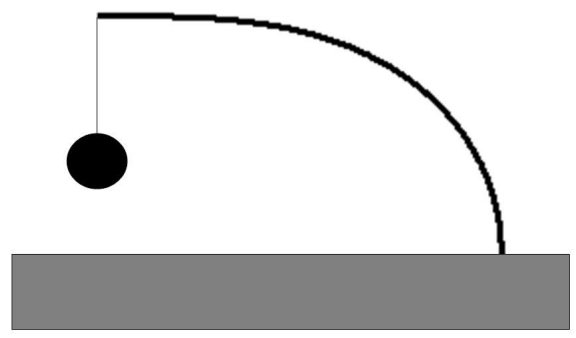

Figure 1. Elastica studied by James Bernoulli

\subsection{James Bernoulli and Elastica}

After James Bernoulli proposed the elastica problem, he discovered that the force of the elastica is proportional to its curvature. Then he found that the arclength and $Y$-component of the elastica having the shape illustrated in Fig.1 can be written as elliptic integrals

$$
s=\int_{X}^{1} \frac{\mathrm{d} X}{\sqrt{1-X^{4}}}, \quad Y=\int_{X}^{1} \frac{X^{2} \mathrm{~d} X}{\sqrt{1-X^{4}}} .
$$

Roughly speaking, we refer to elliptic integrals $[64,70,81]$ when the denominator of their integrand are squire roots of polynomials of the third or the forth order with respect to the integration variable, except some special cases. One should note also that (3) is related to the lemniscate problem and that will be discussed in Section 2.9.

\subsection{Daniel Bernoulli and Elastica: Minimal Principle}

After James Bernoulli left this world in 1705, his brother Johann Bernoulli (16671748) succeeded his academic standing. As Euler's father was a friend of Johann, Euler and Johann's son, Daniel (1700-1782) were pupils of Johann. More details can be found in the books by Bell [2] and Fellmann [20].

Daniel Bernoulli wrote a letter to Euler that elastica is realized when the functional of $Z$

$$
\mathcal{E}[Z]=\int k^{2} \mathrm{~d} s
$$


is minimized. Equation (4) is known as the Euler-Bernoulli energy functional due to Euler's contribution which will be mentioned below. There is no doubt that this statement influenced the step from the least principle of optics by Fermat (1601$1665)$ to the least principle of mechanics. In fact, when one regards $s$ as a time coordinate, $k=\partial_{s} \phi$ could be considered as a velocity and then (4) corresponds to the kinetic energy.

Further this idea is extended to the Dirichlet principle, due to Dirichlet (18051859), which plays a crucial role in the discovery of Riemann surfaces in 1856 by Riemann (1826-1866) [35]. More directly, the Willmore functional is a natural extension of the Euler-Bernoulli functional to a surface in the three dimensional euclidean space $\mathbb{E}^{3}$ which is given by $\int H^{2} \mathrm{~d} v o l$, for the mean curvature $H$ [82]. Both properties are quite similar, see e.g., [47,62].

\subsection{Euler and Elastica: Method}

Euler wrote a book called "Methodus..." in 1744 [19]. There he formulated the variational method and essentially solved the elastica problem in its Appendix, De Curvis Elasticis. By solving the variational problems, he showed that every elastica $Z(s):=X(s)+\sqrt{-1} Y(s)$ is given by

$$
s=\int \frac{\lambda^{2} \mathrm{~d} X}{\sqrt{\lambda^{4}-\left(\alpha+\beta X+\gamma X^{2}\right)^{2}}}, \quad Y=\int^{X} \frac{\left(\alpha+\beta X+\gamma X^{2}\right) \mathrm{d} X}{\sqrt{\lambda^{4}-\left(\alpha+\beta X+\gamma X^{2}\right)^{2}}}
$$

and illustrated them as presented in Fig.2. The sketches were drawn by numerical computations of elliptic integrals for representative points of the elastic curves. By drawing the sketches, he completely classified the shapes of elastica into nine classes. After giving the modern proof of (5) formulated here as Theorem 9, we will add some comments on the nine classes in Section 2.8.

\subsection{Derivation of Euler's Elastica from a Modern Point of View I}

In the following subsections $2.5-2.7$, we will give the modern proof of Euler's formula (5) using the Goldstein-Petrich method [24]. In this subsection, using the Goldstein-Petrich method, we have the static modified Korteweg-de Vries equation $(\mathrm{SMKdV})$ and its relation to the elliptic curve. The infinitesimal variation of elastica $Z(s)$ is denoted by

$$
Z^{\prime}=Z+\left(U_{1} \mathbf{t}+U_{2} \mathbf{n}\right) \delta t .
$$




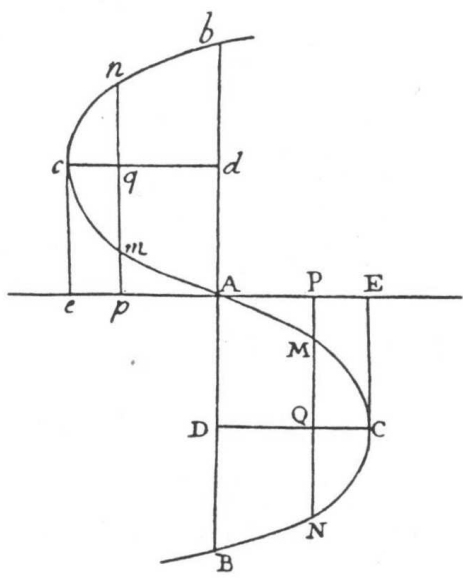

3a. Class 2

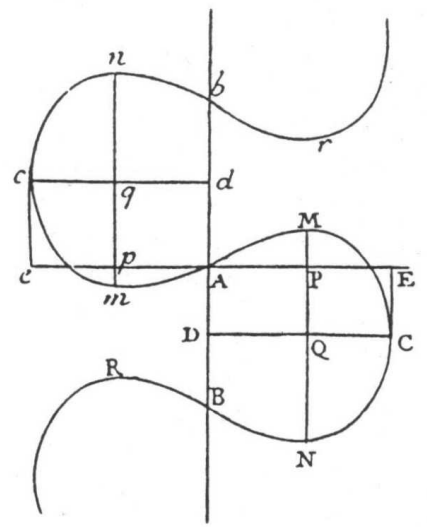

3b. Class 4

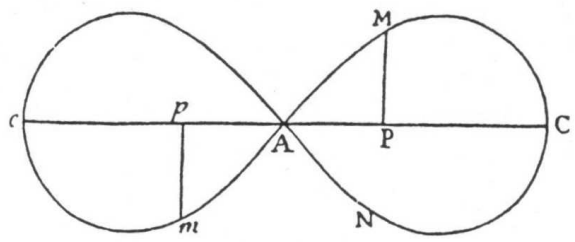

3c. Class 5

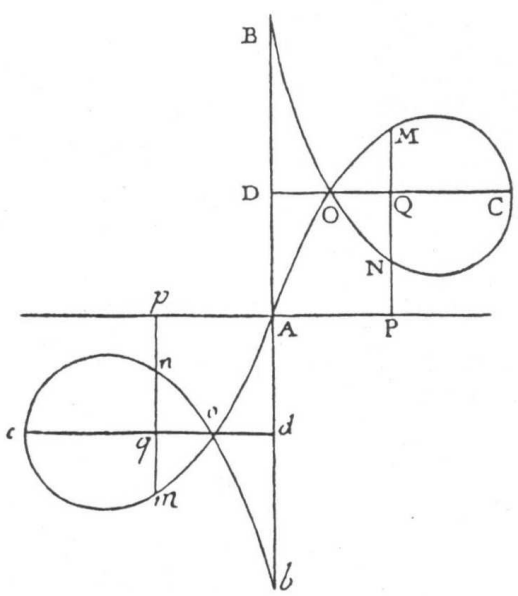

3d. Class 6

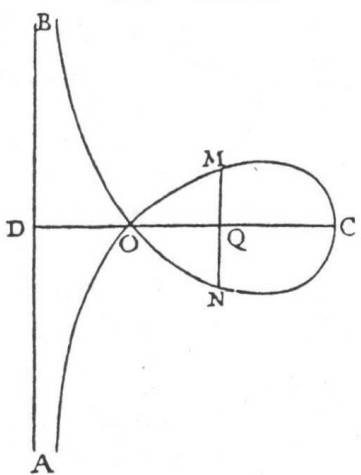

3e. Class 7

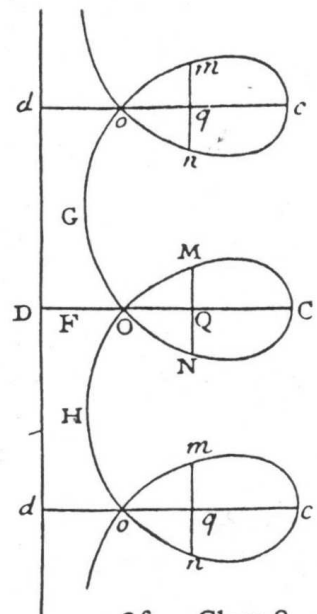

3f. Class 8 
Here $\delta t$ is not the actual time but is considered as an infinitesimal mathematical deformation parameter. However by interpreting it as a mathematical time, $\left(U_{1}, U_{2}\right)$ behaves like a velocity. In other words, by regarding $\left(Z^{\prime}-Z\right) / \delta t$ as $\partial Z / \partial t$, the above relation is rewritten as

$$
\partial_{t} Z=\left(U_{1}+\sqrt{-1} U_{2}\right) \mathrm{e}^{\sqrt{-1} \phi} .
$$

The variation which does not change its local arclength has the property

$$
\left[\partial_{t}, \partial_{s}\right]=0
$$

where $[A, B] \equiv A B-B A$. This property is called the non-stretching condition, which is the same as the compatibility condition in the study of the nonlinear integrable system [39].

The computation of $\left[\partial_{t}, \partial_{s}\right] Z=0$ proves that both

$$
\begin{aligned}
\partial_{t} \partial_{s} Z & =\partial_{t} \mathrm{e}^{\sqrt{-1} \phi}=\sqrt{-1} \partial_{t} \phi \partial_{s} Z \\
\partial_{s} \partial_{t} Z & =\partial_{s}\left(\left(U_{r}+\sqrt{-1} U_{i}\right) \partial_{s} Z\right) \\
& =\left(\left(\partial_{s} U_{r}-k U_{i}\right)+\sqrt{-1}\left(\partial_{s} U_{i}+k U_{r}\right)\right) \partial_{s} Z
\end{aligned}
$$

are equivalent as real functions. Thus the following lemma holds.

Lemma 1. The non-stretching condition (7) is reduced to

$$
\partial_{t} \phi=\partial_{s} U_{2}+k U_{1}, \quad 0=\partial_{s} U_{1}-k U_{2} .
$$

By differentiating the first relation in (8) with respect to $s$ again and substituting the second relation into the first one, the formar is reduced to the following proposition.

Proposition 2. For a deformation, $\partial_{t} Z=\left(U_{1}+\sqrt{-1} U_{2}\right) \mathrm{e}^{\sqrt{-1} \phi}$, the non-stretching condition means

$$
\partial_{t} k=\partial_{s}\left(k+\partial_{s} k^{-1} \partial_{s}\right) U_{1}
$$

Here we have used $\partial_{s} \partial_{t} \phi=\partial_{t} \partial_{s} \phi=\partial_{t} k$. Accordingly the deformation preserving the local arclength must satisfy (9).

Proposition 3. With the non-stretching condition, the variational problem for the energy functional (4) is reduced to

$$
-\frac{\delta \mathcal{E}}{\delta t U_{1}(s)}=\partial_{s}\left(k^{2}\right)+2 \partial_{s} \frac{\partial_{s}^{2} k}{k}=0
$$


or

$$
a k+\frac{1}{2} k^{3}+\partial_{s}^{2} k=0
$$

where $a$ is a real constant. More precisely the differential of equation (11) is known as a static case of the modified Korteweg-de Vries (SMKdV) equation in modern soliton theory.

Proof: The left hand side of (10) is equal to

$$
\lim _{U(s) \delta t \rightarrow 0} \frac{\mathcal{E}\left[Z^{\prime}\right]-\mathcal{E}[Z]}{\delta t U_{1}(s)}=0
$$

and then we obtain the right hand side. By integrating the first equation again and multiplying by $k$, it becomes (11). Due to the reality condition of $\phi$, the parameter $a$ is a real constant number.

Finally we have the governing equation of the elastica:

Proposition 4. For a real constant b, the elastica obeys the equation

$$
\left(\partial_{s} k\right)^{2}+\frac{1}{4} k^{4}+a k^{2}+b=0 .
$$

Proof: By multiplying (11) by $\left(\partial_{s} k\right)$ and integrating it, (11) becomes (12). Here $b$ is an integration constant. Due to the reality, $b$ must be also real.

Remark 5. When one regards $\left(\partial_{s} k, k\right)$ as a point in $(\xi, \eta)$-plane, (12) corresponds to an algebraic curve in $(\xi, \eta)$-plane

$$
\xi^{2}=-\frac{1}{4} \eta^{4}-a \eta^{2}-b
$$

This curve is an elliptic curve and will be denoted by $C_{1}^{\prime}$ in this article.

\subsection{Elliptic Functions}

Here we will give some details about elliptic functions. Let us recall that the coordinates of the point on a circle given by $x^{2}+y^{2}=1$ can be expressed as $(x, y)=(\cos \theta, \sin \theta)$. Noting also that $\mathrm{d} \cos \theta / \mathrm{d} \theta=-\sin \theta$, and we have the relations

$$
-y \frac{\mathrm{d} \theta}{\mathrm{d} x}=1, \quad \frac{\mathrm{d}}{\mathrm{d} x} y \frac{\mathrm{d} \theta}{\mathrm{d} x}=0 .
$$


It should be noted that it is essential that the trigonometric function is interpreted as the inverse function $x(\theta)$ of the solution $\theta(x, y)$ of the differential equations (14). A generalization of this interpretation corresponds to elliptic functions and elliptic integrals. For later convenience, instead of $C_{1}^{\prime}$, we introduce the elliptic curve $C_{1}$

$C_{1}:=\left\{(x, y) ; y^{2}=x^{3}+\lambda_{2} x^{2}+\lambda_{1} x+\lambda_{0}=\left(x-e_{1}\right)\left(x-e_{2}\right)\left(x-e_{3}\right)\right\} \cup\{\infty\}$.

Similar to (14), we characterize the parameter $u$ along the curve $C_{1}$ by

$$
2 y \frac{\mathrm{d} u}{\mathrm{~d} x}=1, \quad \frac{\mathrm{d}}{\mathrm{d} x} y \frac{\mathrm{d} u}{\mathrm{~d} x}=0 .
$$

The solution of (16) is an elliptic integral of the first kind

$$
u=\int_{\infty}^{x} \mathrm{~d} u, \quad \mathrm{~d} u=\frac{\mathrm{d} x}{2 y} \equiv \frac{\mathrm{d} x}{2 \sqrt{x^{3}+\lambda_{2} x^{2}+\lambda_{1} x+\lambda_{0}}}
$$

and the inverse function $x(u)$ of (17) is an elliptic function. If we make $\lambda_{2}=0$ and $y \equiv \bar{y} / 2, x(u)$ is the so called Weierstrass $\wp$-function [81].

Here it should be noted that functional value of the elliptic function $x$ is simply the $x$-component of the graph (curve) $C_{1}$ in the $(x, y)$-plane (affine plane) and $u$ is merely a solution of the linear differential equation (16). For example, the derivative in $u$ can be replaced with the algebraic differential $x$ i.e.,

$$
\frac{\mathrm{d}}{\mathrm{d} u} \equiv 2 y \frac{\mathrm{d}}{\mathrm{d} x} \text {. }
$$

Of course, it is quite useful to regard $\wp$ as an inverse function of an elliptic integral but it is important also to understand that many relations among elliptic functions can be directly obtained as algebraic relations over the commutative ring $\mathbb{C}[x, y] /\left(y^{2}-x^{3}-\lambda_{2} x^{2}-\lambda_{1} x-\lambda_{0}\right)$ and various relations in the linear space of (16).

For later convenience, we also introduce the Legendre relation and the differential of the second kind, which is directly related to the Weierstrass $\zeta$ function. As the trigonometric functions have a single period $2 \pi$, the elliptic functions, e.g., $\wp$ have double periodicity $\left(2 \omega^{\prime}, 2 \omega^{\prime \prime}\right)$

$$
\omega^{\prime}:=\int_{\infty}^{\left(e_{1}, 0\right)} \mathrm{d} u, \quad \omega^{\prime \prime}:=\int_{\infty}^{\left(e_{3}, 0\right)} \mathrm{d} u
$$

which are known as the complete elliptic integrals of the first kind. The differential of the second kind is one form over $C_{1}$ with a singularity and without any residual 
coefficient, whose normalized form is given by

$$
\mathrm{d} r=\frac{x \mathrm{~d} x}{2 y}
$$

which also has a complete elliptic integral of the second kind

$$
\eta^{\prime}=\int_{\infty}^{\left(e_{1}, 0\right)} x \mathrm{~d} u, \quad \eta^{\prime \prime}=\int_{\infty}^{\left(e_{3}, 0\right)} x \mathrm{~d} u .
$$

By computing

$$
\int_{\partial C_{1}^{o}} r \mathrm{~d} u, \quad r:=\int_{\infty}^{(x, y)} \mathrm{d} r
$$

where $C_{1}^{o}$ is a standard parallelogram expression of $C_{1}$, we have the relation due to Legendre (1752-1833), which can be considered as the symplectic structure on the elliptic curve.

Proposition 6. (Legendre relation)

$$
\omega^{\prime} \eta^{\prime \prime}-\omega^{\prime \prime} \eta^{\prime}=\frac{\pi}{2}
$$

As mentioned above, $\left(\wp(u), \partial_{u} \wp(u) / 2\right)$ should be identified with $(x, y)$ of $u=$ $\int_{\infty}^{(x, y)} \mathrm{d} u$. There exists an entire function $\sigma$ over $\mathbb{C}$ such that

$$
\zeta(u)=\frac{\partial}{\partial u} \log \sigma(u), \quad \wp(u)=-\frac{\partial^{2}}{\partial u^{2}} \log \sigma(u) .
$$

Here $\sigma$ is the Weierstrass sigma function

$$
\begin{gathered}
\sigma(u)=\frac{2 \omega^{\prime}}{2 \pi \sqrt{-1}} \exp \left(\frac{\eta^{\prime} u^{2}}{2 \omega^{\prime}}\right) \frac{\theta_{1}\left(u / \omega^{\prime}\right)}{\theta_{1}^{\prime}(0)} \\
\theta_{1}(v)=\sqrt{-1} \sum_{n=-\infty}^{\infty} \exp \left(\sqrt{-1} \pi\left(\tau(n-1 / 2)^{2}+(2 n-1)(v+1)\right)\right) .
\end{gathered}
$$

We have an identity between $\zeta$-function and an integral of the second kind

$$
\zeta(u)=-\int_{\infty}^{(x, y)} \mathrm{d} r=-\int_{\infty}^{(x, y)} x \mathrm{~d} u .
$$




\subsection{Derivation of Euler's Elastica from a Modern Point of View II}

Let us return to the derivation of Euler's results of elastica. In other words, let us consider a connection of $(x, y)$ with the above elastic curve $C_{1}^{\prime}$. Let us identify $u$ and $s$.

Than

$$
\begin{aligned}
x & :=\frac{1}{4} \sqrt{-1} \partial_{s} k+\frac{1}{8} k^{2}+\frac{1}{12} a \\
y & :=\frac{1}{2} \partial_{s} x=\frac{1}{2}\left[\sqrt{-1}\left(\frac{1}{8} k^{3}+\frac{1}{4} a k+\frac{1}{4} \sqrt{-1} k \partial_{s} k\right)\right] .
\end{aligned}
$$

We note that the correspondence between $x$ and $k$ is known as the Miura transformation [39] which connects the Korteweg-de Vries ( $\mathrm{KdV})$ equation and the modified $\mathrm{KdV}(\mathrm{MKdV})$ equation. In this way equation (12) is reduced to

$$
y^{2}=\left(x+\frac{1}{6} a\right)\left(x-\frac{1}{12} a-\frac{1}{4} \sqrt{b}\right)\left(x-\frac{1}{12} a+\frac{1}{4} \sqrt{b}\right) .
$$

Let $e_{1}=-\frac{1}{6} a, e_{2}=\frac{1}{12} a+\frac{1}{4} \sqrt{b}$, and $e_{3}=\frac{1}{12} a-\frac{1}{4} \sqrt{b}$. For later convenience, we also assume that $a^{2}-b=16$. We now identify the Weierstrass $\wp$ function and $x$ up to its origin, i.e., $x(s)=\wp\left(u+s_{0}\right)$ for $s_{0} \in \mathbb{C}$, as mentioned above.

The relations (22) becomes

$$
\sqrt{-1} k=\frac{2 y}{x-e_{1}}=\frac{\partial_{s} x}{x-e_{1}}
$$

and lead us to the following theorem

Theorem 7. By choosing the origin of angle $\phi$ and $s_{0}$ we have

$$
\begin{gathered}
\partial_{s} Z \equiv \mathrm{e}^{\sqrt{-1} \phi}=\sqrt{-1}\left(x(s)-e_{1}\right) \\
Z=\sqrt{-1}\left(-\zeta\left(s+s_{0}\right)-e_{1} s\right)+Z_{0} .
\end{gathered}
$$

Proof: The tangential angle of the elastica is given by

$$
\phi(s)=\frac{1}{\sqrt{-1}} \log \left(x(s)-e_{1}\right)+\phi_{0} .
$$

It means that the tangential vector of elastica is represented by an elliptic function and using the $\zeta$ function, we have an explicit formula of $Z$. In other words, it is found that $k \equiv \partial_{s} \phi$ in (22) satisfies (11) and (12) and vice versa. 
Remark 8. We should note also that (23) means

$$
\mathrm{d} Z=\sqrt{-1} \frac{x \mathrm{~d} x}{2 y}-\sqrt{-1} e_{1} \frac{\mathrm{d} x}{2 y}
$$

where the first term is regarded as the differential of the second kind and the second term as the differential of the first kind. Using the Weierstrass sigma function, we have the identity

$$
\sqrt{-\partial Z}=\frac{\mathrm{e}^{-\eta^{\prime} u} \sigma\left(u+\omega^{\prime}\right)}{\sigma\left(\omega^{\prime}\right) \sigma(u)} .
$$

Then we have the Euler's expressions

Theorem 9. (Euler's expression) By choosing its appropriate origin of $Z$, we have

$$
s=\int^{X} \frac{\lambda^{2} \mathrm{~d} X}{\sqrt{\lambda^{4}-\left(\alpha+\beta X+\gamma X^{2}\right)^{2}}}, \quad Y=\int^{X} \frac{\left(\alpha+\beta X+\gamma X^{2}\right) \mathrm{d} X}{\sqrt{\lambda^{4}-\left(\alpha+\beta X+\gamma X^{2}\right)^{2}}} .
$$

Proof: We note the relation $1=-\left(e_{3}-e_{1}\right)\left(e_{2}-e_{1}\right)$ since $a^{2}-b=16$ or $\left|e_{1}-e_{2}\right|=\left|e_{1}-e_{3}\right|=1$. Let us employ the Euler representation, $\mathrm{e}^{\sqrt{-1} \phi(s)}=$ $\cos \phi(s)+\sqrt{-1} \sin \phi(s)$, i.e., $\left(\partial_{s} X, \partial_{s} Y\right)=(\cos \phi(s), \sin \phi(s))$. We have identified $x\left(s-s_{0}\right)$ with $\wp(s)$ for appropriate $s_{0} \in \mathbb{C}$. The Weierstrass $\wp(s)$ function satisfies

$$
\begin{aligned}
\wp\left(u-\omega^{\prime}\right)-e_{1} & =\frac{\left(e_{2}-e_{1}\right)\left(e_{3}-e_{1}\right)}{\left(\wp(u)-e_{1}\right)} \\
\wp\left(u-\omega^{\prime}\right)-\wp(u) & =\frac{1}{2} \frac{\mathrm{d}}{\mathrm{d} u} \frac{\wp^{\prime}(u)}{\left(\wp(u)-e_{1}\right)} .
\end{aligned}
$$

The former one is obtained due to duality between the zero and the pole and the latter one is due to the addition theorem [81]. These formulas generate the relation

$$
\cos \phi(s)=\left(\sqrt{-1}\left(x-e_{1}\right)+1 / \sqrt{-1}\left(x-e_{1}\right)\right) / 2=\partial_{s} k / 4
$$

which leads us to

$$
X(u)=X_{0}+\frac{1}{4} k(u)
$$

for an appropriate origin $X_{0} \in \mathbb{R}$. Hence this relation with (12) means fact that $\left(X(s), \partial_{s} X(s)\right)$ satisfies the algebraic relation

$$
\left(\partial_{s} X\right)^{2}=-\left(4\left(X-X_{0}\right)^{4}+a\left(X-X_{0}\right)^{2}+b / 16\right) .
$$


The right hand side is equal to $\left(a^{2}-b\right) / 16-\left(2 X^{2}+a / 4\right)^{2}$ by letting $X_{0}$ vanish. Noting that $a^{2}-b=16$, this equation implies that the arclength $s$ is given by

$$
s=\int^{X} \frac{\mathrm{d} X}{\sqrt{1-\left(2 X^{2}+a / 4\right)^{2}}} \cdot
$$

The first relation in Theorem 9 is essentially proved. Further since we have

$$
\partial_{s} Z-\partial_{s} X=\sqrt{-1}\left(x-e_{1}\right)-\sqrt{-1} \frac{1}{4} \partial_{s} k=\frac{1}{8} k^{2}+\frac{1}{4} a
$$

the $Y$-component is given by

$$
Y=\int^{X} \frac{\left(2(X)^{2}+a / 4\right) \mathrm{d} X}{\sqrt{1-\left(2 X^{2}+a / 4\right)^{2}}} .
$$

These essentially recover Euler's results.

By changing $\alpha, \beta, \gamma$, and $\lambda$, Euler classified the elastica as shown in Fig.2. Corresponding to $\alpha, \beta, \gamma$, and $\lambda$, the variation of $a$ and $b$ classifies the elastica with the condition $a^{2}-b=16$. It implies that the dimension of the parameter space is one as shown in (32) for the classification with respect to similarity of shapes.

In the computation of elastica, the condition that $\phi$ and $s$ are real is necessary. We call the condition reality condition i.e., $\left|\partial_{s} Z\right|=1$ and $s$ is real.

Let us call the tangential period $\hat{\omega}$ of the (open) elastica that satisfies

$$
\partial_{s} Z(s+\hat{\omega})=\partial_{s} Z(s)
$$

Further we define an index of (open) elastica by

$$
\operatorname{index}\left(\partial_{s} Z\right)=\frac{1}{2 \pi \sqrt{-1}}\left(\log \partial_{s} Z(s+\hat{\omega})-\log \partial_{s} Z(s)\right) .
$$

Here we give a formula of the Euler-Bernoulli energy function

Proposition 10. The Euler-Bernoulli energy of elastica with unit length as its tangential period is given by

$$
E=8 \frac{\zeta(\hat{\omega})}{\hat{\omega}}-\frac{1}{3} a=8 \frac{\zeta(\hat{\omega})}{\hat{\omega}}+2 e_{1} .
$$

Proof:

$$
\frac{1}{2} \int_{\hat{\omega}} k^{2} \mathrm{~d} s=4 \int_{\hat{\omega}}\left(\frac{1}{8} k^{2} \mathrm{~d} s+\frac{1}{4} \sqrt{-1} \partial_{s} k\right)=4 \int_{\hat{\omega}} x \mathrm{~d} s-\frac{1}{3} a \hat{\omega} .
$$


By considering the energy $E=E(\ell)$ as a function of the length, we have the relation

$$
\frac{1}{\hat{\omega}} E(\hat{\omega})=E(1)
$$

which gives the relation.

The number $\tau:=\omega^{\prime \prime} / \omega^{\prime}$ is a complex number called modulus, which determines the elliptic curve uniquely modulo trivial transformation, translation, dilatation and so on, and also determine the shape of the elastica.

Due to the reality condition, we list the classification of elastica as follows, and for proofs refer to $[52,60]$

Proposition 11 (cf. $[17,52,60,66]) \quad 1$. For the case $-4<a \leq 4, s_{0}=\omega^{\prime \prime}$, $\hat{\omega}=4 \omega^{\prime}-2 \omega^{\prime \prime} \in \mathbb{R}, E=8 \frac{2 \eta^{\prime}-\eta^{\prime \prime}}{2 \omega^{\prime}-\omega^{\prime \prime}}+2 e_{1}, \tau \in \sqrt{-1} \mathbb{R}_{>0} \cup\left(\frac{1}{2}\right)$ and index $\left(\partial_{s} Z\right)$ vanishes.

2. For $4<a, s_{0}=\frac{\omega^{\prime \prime}+\omega^{\prime}}{2}, \hat{\omega}=2 \omega^{\prime \prime} \in \mathbb{R}, E=8 \frac{\eta^{\prime \prime}}{\omega^{\prime \prime}}+2 e_{1}, \tau \in \sqrt{-1} \mathbb{R}_{>0}$, and $\operatorname{index}\left(\partial_{s} Z\right)$ is equal to \pm 1 .

Here $\mathbb{R}_{>0}$ is $\{x \in \mathbb{R} ; x>0\}$.

Due to the reality condition of the elastica, the moduli $\Lambda$ of the elastica are given by [60]

$$
\Lambda:=\sqrt{-1} \mathbb{R}_{>0} \cup\left(\frac{1}{2}+\sqrt{-1} \mathbb{R}_{>0}\right) \cup \infty \text { modulo } \operatorname{PSL}(2, \mathbb{Z})
$$

as a subspace of the moduli of the elliptic curves, $\Lambda \subset \mathbb{H} / \operatorname{PSL}(2, \mathbb{Z})$, where $\mathbb{H}$ is the upper half plane, i.e., $\mathbb{H}:=\{z \in \mathbb{C} ; \operatorname{Im} z>0\}$.

\subsection{Classification of Euler's Elastica From a Modern Point of View}

Theorem 9 contains all possible shapes of the elastica. Further by extending its coefficients to complex numbers $X(s)$ contains all kinds (moduli) of elliptic functions.

In respect to Euler's sketches, we will mention the classification given in Proposition 11, and the individual classes in Fig.2. It should be noted that in [17,52], the classification is the same as the solutions of the oscillating pendulum problem and Sachkov recently gave more precise classifications of the elastica in [66].

1. Class $1(a=-4)$ : The first class corresponds to the straight line. 


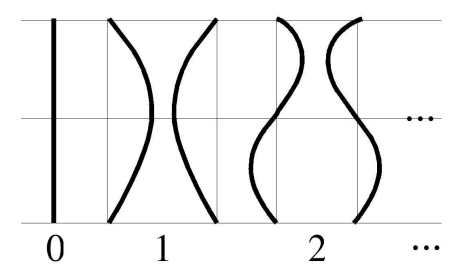

Figure 3. The bifurcation sequences of elastic curves.

2. Class $2(a \in(-4,0))$ : The Class 2 is related to a bifurcations and a symmetry breaking. When we push both sides of a thin elastic plate, we might meet the shape in the Class 2. Depending on the forces and so on, there appear two or three nodes and it swells to right or left hand side. Topologically this picture is connected with the orientation of the curve, or homotopy group $\pi_{0}(\mathrm{O}(2, \mathbb{R}))=\mathbb{Z}_{2}=\{0,1\}$. It means that elastica is a nice prototype of such a bifurcation and a symmetry breaking, which is classified by the integer $\pm n, n \geq 0$. Actually $n+1$ is the number of crossing of $y$-axis for $n \geq 1$.

3. Class $3(a=0)$ : The class 3 discovered by James Bernoulli, which is closely related to lemniscate which will be discussed in Section 2.9. By letting $(s, X, Y)$ in Theorem 9 be written as $(s, X, Y)=:(\hat{s} / \sqrt{2}, \hat{X} / \sqrt{2}, \hat{Y} / \sqrt{2})$, the formulas in Theorem 9 become that ones in (3).

4. Class 2, 3, $4\left(a \in\left(-4, a_{\infty}\right)\right), a_{\infty}:=2.60891826 \cdots:$ We encounter similar shapes of the class 2, 3, and 4, when we handle a paper tape, a carpet, an electric line and so on. Of course, their actual shapes depend upon their thickness and twist but sometimes are approximated by the shapes in these classes. This fact comes from the naturalness of elliptic functions and integrals $[64,70]$.

5. Class $5\left(a=a_{\infty}\right)$ : The Class 5 consists of the mysterious figure eight, which is related to the lemniscate (see Section 2.9). The modulus of the class 5 is $\tau=0.704761 \cdots \times \sqrt{-1}+1 / 2$. For a certain transformation, we have another modulus $\hat{\tau}=(1-2 \tau)^{-1}=0.70946 \cdots \times \sqrt{-1}$. As $\hat{\omega}=4 \omega^{\prime}-2 \omega^{\prime \prime}$ is the tangential period of the loop the closed condition is $Z\left(s+2\left(2 \omega^{\prime}-\omega^{\prime \prime}\right)\right)=Z(s)$. For the eight shape figure, by choosing an appropriate origin, we have $Z(0)=-Z\left(2 \omega^{\prime}-\omega^{\prime \prime}\right)$. It means that $2 \eta^{\prime}-\eta^{\prime \prime}=$ $e_{1}\left(2 \omega^{\prime}-\omega^{\prime \prime}\right)$ because $\eta^{\prime}=\zeta\left(\omega^{\prime}\right)$ and $\eta^{\prime \prime}=\zeta\left(\omega^{\prime \prime}\right)$. Due to a kind of the 
Landen transformation

$$
\eta^{* \prime}=\eta^{\prime}+e_{1} \omega^{\prime} / 2, \quad \omega^{* \prime}=\omega^{\prime} / 2, \quad e^{*}{ }_{3}=-2 e_{1}
$$

we have $\eta^{\prime}=\omega^{\prime} e_{1} / 2$. Also we find that $K=2 E$ if we make use the Jacobi elliptic integrals because $K=\left(e_{1}-e_{3}\right)^{1 / 2} \omega^{\prime}$ and $E=\left(e_{1}-e_{3}\right)^{-1 / 2}\left(\eta^{\prime}+\right.$ $\left.e_{1} \omega^{\prime}\right)$ [81]. As $\mathrm{e}^{-\eta^{\prime} \omega^{-1} u^{2} / 2}$ is the bilinear factor of the Weierstrass sigma function, it becomes $\mathrm{e}^{-e_{2} u^{2} / 2}$ for the figure eight case.

The shape of the Class 5 is realized by twisting of the circle in Class 9 . For example, we appropriately connect the ends of the string of the guitar and twist it. What shape we obtain if we twist it again? Of course, the actual string forms a strange shape which is not in the classification table of elastica in a plane. In other words, there are only two type closed loops in the classification table, which is closely related to the fact that we implicitly deal with $\mathbb{Z}_{2}$-symmetry.

6. Classes 6, 7, $8\left(a \in\left(a_{\infty}, \infty\right)\right)$ : These also appear as shape of electric lines and loops like in Class 4. However it should be noticed that Classes 7 and $8(a \in[4, \infty))$ have indices \pm 1 whereas the 6-th Class $\left(a \in\left(a_{\infty}, 4\right)\right)$ has vanishing index.

7. Class $7(a=4)$ : We will comment on the 7-th Class and its relation to the soliton theory later on. Here we will mention that this shape was rediscovered as a loop soliton by Konno, Ichikawa and Wadati in 1979 [37] by considering soliton equations, integrable nonlinear partial differential equations. The loop soliton is given so that the half of its curvature satisfies the MKdV equation [30]. It is known that all of shapes in Fig.2 can be regarded as elliptic solutions of the loop soliton. When we play with a rope or lasso, a twisted rope sometimes has a loop which moves along the asymptotic direction of the center axis. Its shape is the loop soliton with the shape, Class 7. The loop solitons are also closely related to the statistical mechanics of elasticas as we see. All these means that the elastica problem is a prototype of the soliton equations obtained in 1950's-1980's after two hundred years passed since 1744 .

8. Class $9(a=\infty)$ : Class 9 consists of the circle $X^{2}+Y^{2}=1$ whose index is \pm 1 . 


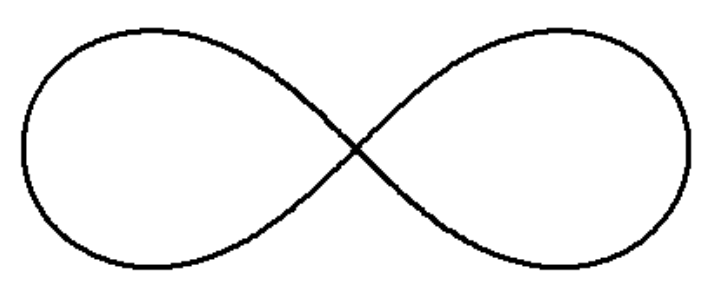

Figure 4. Lemniscate curve.

\subsection{Lemniscate}

Let us digress a little bit and give some comments on the lemniscate problem and a history of elliptic functions [80]. The lemniscate curve drawn in Fig.4 was also proposed by James Bernoulli, and can be presented as the algebraic relation

$$
\left(X^{2}+Y^{2}\right)^{2}=X^{2}-Y^{2} \text {. }
$$

The arclength of the lemniscate is expressed by the formula (3) (see also [64]).

Now let us look at two figures eight, Fig.4 and Class 5 in Fig.2. Whereas the shape of elastica is given by transcendental function $\zeta(s)$ the lemniscate is defined by the algebraic relation. It is not clear whether James Bernoulli recognised or not but both his proposed problems have contributed to the development of the elliptic function theory.

Fagnano (1682-1766) had studied algebraic properties of some integrals since 1714 and in this way become interested in lemniscate. He found some "additive" relation of (3) and submitted a paper to the Academy. Euler received it as a referee on December 23, 1751 and have extended Fagnano's studies to more general ones. In this connection Jacobi (1804-1851) stated that this day is the birthday of the elliptic functions [81].

Gauss (1777-1855) called the inverse function of the integral (3) a lemniscate function in 1796 and started its study in secret. He had found the addition formula and so on for the lemniscate function [28, 35, 80]. Later on Abel (1802-1829) and Hurwitz (1859-1919) have discovered other number theoretic properties of the lemniscate function [29].

The lemniscate played the central roles in the history of the elliptic functions, especially of their algebraic properties. However it should be noted that Houzel 
(p.473 in [28]) stated that Gauss would have considered the lemniscate function by looking over the figures of Euler's elasticas in Fig.2.

The transformation between the lemniscate and elastica of James Bernoulli Fig.1 are given by the relation between their tangential angles [44]

$$
\phi_{\text {lemn }}=\frac{3}{2} \phi_{\text {elas }} .
$$

Here both $\phi$ 's with accessory should be naturally interpreted. Since the tangential angle in Fig. 1 crosses perpendicularly with both the $X$ and the $Y$-axes, $\left(\phi_{\text {elas, } X}=\right.$ $\left.\pi / 2, \phi_{\text {elas }, Y}=\pi\right)$, in the first quarter region, i.e., $\{(X, Y) ; X \geq 0, Y \geq 0\}$, the correspondence means that the lemniscate curve should crosses the $Y$-axes at $5 / 4 \pi,\left(\phi_{\operatorname{lemn}, X}=\pi / 2, \phi_{\operatorname{lemn}, Y}=5 \pi / 4\right)$, and recovers the relation

$$
\left(\phi_{\text {lemn }, X}-\phi_{\text {lemn }, Y}\right)=3\left(\phi_{\text {elas }, X}-\phi_{\text {elas }, Y}\right) / 2 .
$$

As the lemniscate curve in the first quarter region should be symmetrically considered, it must be closed. Since the manipulation does not have any effects on the arclength, the arclength is given by the same formula.

It is also interesting because $3 / 2$ reminds us about the spin of the supergravty.

Though it is not clear whether James Bernoulli recognised the relations among the two figures-eight, he had considered both shapes and the same elliptic integral as their arc lengths.

\subsection{Euler's Elastica from a Historical Point of View}

First, we will emphasize that the $X$-component in Fig.2 is a periodic function of the arclength $s$. As an inverse function of elliptic integral it is called elliptic function $[64,70,81]$ and the inverse function $X(s)$ of the elliptic integral $s(X)$ in (5) is an elliptic function of the arclength $s$. This fact is very important from a viewpoint of the history of elliptic functions.

As mentioned, before that Gauss have initiated the study of the lemniscate function. In 1824, the paper by Abel appeared where he stated that the inverse function of the elliptic integral is important and leads to the introduction of the present day elliptic functions. Of course, the works by Jacobi, Riemann, Weierstrass (18151897) and so on were indispensable for establishments of the so-called elliptic function theory. Ordinarily, from the viewpoint of importance of the inverse function, it is usually regarded that the date of discovery of the elliptic function is 1796 (Gauss) or 1824 (Abel) [28] contrary to the statement of Jacobi. 
Let us return to the elastica problem. When we look back at a historical event, we should not interpret it without considering the historical background neither analyze it using our modern common sense. However the first remarkable point is that Euler gave illustrations and that the $X$-components are expressed by periodic functions of the individual arclength $s$.

One can ask the question: Could Euler notice the fact? If he had done, it means that he would have considered the inverse function of (5). In other words, it implies that he would have looked elliptic functions fifty years before Gauss's discovery, thirteen years before the birth day Jacobi said. Houzel (p.473 in [28]) pointed out that Euler could not but, as mentioned above, Gauss would have considered the lemniscate function by looking over Euler's figures.

Even though Houzel's opinion is right, we should say that the studies of Euler was beyond the studies of his time. He discovered the most of the results in the elliptic function theory. In fact he also discovered the special case of the Legendre relation in Proposition 6,

$$
\text { Height in Fig. } 1 \times \text { Arclength of Fig. } 1=\int_{0}^{1} \frac{X^{2} \mathrm{~d} X}{\sqrt{1-X^{4}}} \int_{0}^{1} \frac{\mathrm{d} X}{\sqrt{1-X^{4}}}=\frac{\pi}{2} .
$$

Further he moved a point in the moduli of elliptic functions, which is the beginning of the study of moduli of elliptic curves.

Of course the most important fact is that by discovery of the variational principle, constructing the variational method and the theory of "elliptic function", the elastica problem was solved completely after about fifty years passed since it had been proposed. "How should we choose the parameters to obtain a certain shape?" "What shape of elastica can be allowed?" Euler basically answered these inquiries. Behind the resolution, he potentially studied the variational problem, a nonlinear differential equation, algebraic geometry of elliptic curve, differential geometry of the plane curves, elliptic integral theory, classification of moduli and so on, and he potentially understood them. (It should be remarkable that if he had not understood the dimension and structure of moduli of elliptic functions, he could not have classified the elastica.) With respect to elliptic function theory, these potential discoveries are seventy years before the proper discoveries in history of elliptic functions. From the viewpoint of the nonlinear integrable theory or soliton theory, Euler's work is even earlier with two hundred years.

Here we should emphasize also that in the works which were started by James Bernoulli, Euler and Bernoulli's did not adhere to their own established methods and strategies. In those days, the theory of primary functions were wellestablished in the practical meaning. Thus one can wonder if it was possible to 
apply the established tools to the elastica problem by peculiar approximation. Indeed, even nowadays, we could find several strange papers on the elastica problem and its variation in which each author use some strange approximations and excuses, and then gave different results from Euler's. However for the purpose to understand the shape of elastica, these studies not hesitate to offer a novel way and strategies to recover the correct answers. Sometimes they produce new tools and new theories to solve the problem. The tools and theories were so sophisticated but they could be applied to many other problems.

\subsection{Beyond Euler's Elastica}

As mentioned above, the elastica problem was solved in a certain sense. Of course, the elastica problem does not finish at all. We will pick up some of its related topics because behind them there exist so many works and studies connected with various aspects of the elastica problem. Readers who are concerned with some of the topics should check the references of the cited papers. According to [41,73], Laplace (1749-1827) and Maxwell (1831-1879) investigated capillary shape problem and have found its relation to the elastica. Kirchhoff (1824-87) studied a generalized problem of elastica, i.e., the elastic rod in the three dimensional space [34], elastic plane, and so on [73]. Born have studied elastic beam in the three dimensional space in his $\mathrm{PhD}$ thesis [41]. Physically it is related to the vortex filament in fluid dynamics. Hasimoto [27] discovered special solutions including Euler's elastica at least partially. Tsuru [75] had found special solutions of elastic rods in three dimensional space. Recently, Fukumoto [21] investigated the relations between vortex filaments and elastic rods. Nishinari [61] partially investigated the dynamics of elastic rod governed by the nonlinear integrable equations. Generalization of embedded space from the euclidean space to more general space was started by Langer and Singer [40,67]. Pinkall also studied it in the framework of the differential geometry [62]. Kawakubo had found more general solutions in several three dimensional spaces [31]. Watanabe considered the heat kernel with Dirichlet and Neumann boundary conditions behind the Euler-Bernoulli energy and its generalization [78].

Bryant and Griffiths [10] have investigated the elastica problem as a variational problem and its generalization from kinematic viewpoints. Arroyo, Barros and Garay $[1,25]$ defined generalized functionals of Euler-Bernoulli energy along the line of the generalization of Chen-Willmore energy to Willmore energy [82], and investigated associated variational problems as harmonic maps and their application to the particle physics. On the other hand, following Maxwell's inves- 
tigation of elastica, Sachkov considered more precise classification of original elastica [66], which gives very beautiful results, and Vassilev, Djondjorov and Mladenov [77] have studied the shapes of the fluid membranes where the pressure term causes a generalization of Euler's elastica. As recently the discretization is essential in the nonlinear integrable system, discretization of elastica was done by Sogo [68]. Other modern investigation of elastica was done by Djondjorov, Hadzhilazova, Mladenov and Vassilev [17]. Mumford [60] recognized the elastica as a problem in computer vision after he had extracted the essentials of elastica as given in Proposition 11.

The loop space itself is still an open problem and Brylinski [11] had studied the problem. Goldstein and Petrich [24] started to study the relations between dynamics of a curve and nonlinear integrable systems in the way similar to Hasimoto's study of the vortex [27]. Following them, Previato investigated its relation to algebraic geometrical objects in [65] and the present author had studied a generalization of the elastica problem in which elastica revives as a statistical mechanics of elastica or quantized elastica.

\section{Quantization of Elastica}

\subsection{Statistical Mechanics of Elasticas}

DNA usually shrinks in a very small space whereas it sometimes enlarges on cell's splitting. The problem arises how it behaves depending upon the circumstances, that is the problem of folding problem. We, of course, have to consider several reasons, biochemical properties, electric properties and so on. However it is a kind of shape problem like elastica. In order to find its complete solution, the knowledge of the shape is necessary.

It is actually a problem how we understand, classify and account the shape of large polymer, such as DNA. Fig. 5 which is a picture of atomic force microscopy (AFM) shows typical shape of the plasmid DNA samples at room temperature [42]. They have the form of a helix with a large number of loops of size over several $10 \mathrm{~nm}$, which is called superhelix or supercoile. The size over several $10 \mathrm{~nm}$ should not be treated merely by chemical effects. In the polymer physics, the entropy causes the crucial effects on the universal behaviour, which are less influenced from micro-(quantum)-effects. The pictures read that there are weak elastic force. However if they are elasticas in a plane, the shapes should be one in the classification table of Euler but they are not. 
There are several considerable reasons why they do not belong to the classification table of Euler in Fig. 2, e.g., chemical effects, polymer physics effects, stretching effects, three dimensional effect, twist effect, and so on. Original elastica is a model of non-stretching thin elastic rod in a plane, whose twist is negligible.

Here we will comment on the studies concerning the extension of elastica in a plane to one in the three dimensional space with the same energy functional (4). Then due to the consideration above, the obtained shapes must be expressed by a rational function of elliptic and trigonometric functions. The obtained shapes might be more complex than Euler's because of the three dimensional effects but they must repeat some simple patterns due to the double periodicity of elliptic functions, which completely differ from those in Fig. 5.

Surely, we should consider effects by which a variety of shapes should be allowed as in Fig. 5. As another effect, by studying such a thermal effect of non-stretching polymers [46], because it is expected that the DNA might be influenced by the temperature since its size is very small. The motivation comes from the fact that as the shape of DNA is more complicated than the shape described by elliptic functions. In fact, due to the thermal effect, we have various shapes and will describe this effect in detail below. Of course, the reason why DNA differs from Euler's might be due to chemical effects but it is very interesting how small elastica is influenced by this. Considering the thermal effects is equivalent to the study

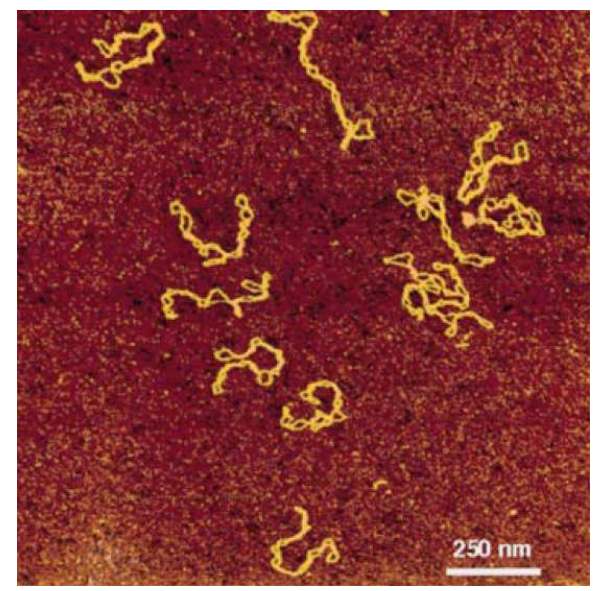

Figure 5. AFM picture of figures of the supercoiled DNA at room temperature.

of the partition function. The problem is to compute the partition function of the 
non-stretching loops at the temperature $T:=1 / \beta$ which is given by

$$
\mathcal{Z}[T]=\sum_{\text {all shapes of } Z} \mathrm{e}^{-\mathcal{E}[Z] / T} .
$$

Here $\mathcal{E}$ is the Euler-Bernoulli energy functional (4). We will call this problem statistical mechanical problem of elastica or quantized elastica problem due to the resemblance between quantum mechanics and statistical mechanics.

The relations between DNA and elastica had been studied for more than four decades (see $[16,32,76]$ and references therein), though the partition function (35) had not be considered explicitly. Kholodenko showed that these problems are closely related to the modern mathematical physics including quantum field theory [32]. Chirikjian attempted to estimate the excluded-volume effect in the conformations.

If one does not impose the non-stretching condition, the statistical mechanical problem becomes easier. This model is known as semiflexible polymer-chain model and there are so many current studies though we will not touch these topics in this article. However it should be noted that the variation with non-stretching condition completely differs from imposition of non-stretching condition after the variational calculus without non-stretching condition. Thus the model does not recover the Euler-Bernoulli elastica when the temperature approaches to zero because the non-stretching condition in Euler's elastica plays the crucial role in the variational calculus. Thus the model is similar but quite different from elastica and quantized elastica. We should emphasize that though elastica is very natural as shown in 2.8 , the model differs from elastica at zero temperature.

\subsection{Quantized Elastica}

Here we will comment on the motivation of studying the quantized elastica from another point of view. It is known that Einstein gravitations, Yang-Mills gauge fields, non-linear sigma model and so on, are classical objects governed by nonlinear equations. When we consider the quantum effect of these fields however it is very difficult to deal with full quantum effect of such geometrical objects. Elastica is the simplest geometrical object that can serve as a toy model which obeys the non-linear equation, SMKdV equation and it is the simplest harmonic problem or nonlinear sigma model. Our study is related to the problems how to deal with the geometrical object in the heat bath and how to quantize it $[46,55,56]$. To solve the problem requires that we have to answer how to classify the geometrical objects with energy but leaves the observation problem as in quantization of 
the geometry like Einstein gravity. It is believed that the quantized elastica problem must be one of prototypes of the quantization of geometry. Further we could check the results using AFM [42].

In order to define the partition function (35) more precisely, we define its domain, moduli of quantized elastica [56]

$$
\mathcal{M}_{\text {elas }}^{\mathbb{C}}:=\left\{Z: S^{1} \rightarrow \mathbb{C} ; \oint \mathrm{d} Z=2 \pi,\left|\partial_{s} Z\right|=1\right\} / \sim
$$

where $\sim$ means the euclidean motion. $\mathcal{M}_{\text {elas }}^{\mathbb{C}}$ has a spectrum decomposition

$$
\mathcal{M}_{\text {elas }}^{\mathbb{C}}:=\coprod_{E} \mathcal{M}_{\text {elas }, E}^{\mathbb{C}}, \quad \mathcal{M}_{\text {elas }, E}^{\mathbb{C}}:=\left\{Z \in \mathcal{M}_{\text {elas }}^{\mathbb{C}} ; \mathcal{E}[Z]=E\right\} .
$$

It is the set of non-stretch loops in real analytic category. As the Dirichlet principle works for the minimal principle [35], even in the loop space with measure and energy given by (4), the principle works weakly. In other words, one can neglect the effect coming from a loop with wild topological behaviour. Highly winding loop does not contribute to the partition function.

Then the partition function (35) becomes [46,56]

$$
\mathcal{Z}[\beta]=\sum_{E} \operatorname{vol}\left(\mathcal{M}_{\text {elas }, E}^{\mathbb{C}}\right) \exp (-\beta E)
$$

which means that the problem to evaluate the partition function is reduced to

1. determination of the equi-energy flow (orbit)

2. evaluation of the volume of the flow (orbit), $\operatorname{vol}\left(\mathcal{M}_{\text {elas }, E}^{\mathbb{C}}\right)$; $\left(\log \operatorname{vol}\left(\mathcal{M}_{\text {elas }, E}^{\mathbb{C}}\right)\right) / \beta$ corresponds to the entropy, and

3. determination of these distribution over $\{E\}=\mathbb{R}_{\geq 0}$, and their sum $\{E\}=$ $\mathbb{R}_{\geq 0}$.

Michor and Mumford recently studied the moduli of loops on a real plane in the category of smooth functions in order to classify the shape of loops [58]. They investigated the minimal points related to energy functional which is modified by the Euler-Bernoulli functional (4). On the other hand, in the quantized elastica problem, the moduli of loops in the category of regular functions are naturally introduced and thus the flow is naturally given via the MKdV hierarchy as follows. In other words, we will mainly consider shape of the quantized elastica as a function of $S^{1}$ belonging to the set of the regular functions over $S^{1}, \mathcal{C}^{\omega}\left(S^{1}, \mathbb{R}\right)$ or $\mathcal{C}^{\omega}\left(S^{1}, \mathbb{C}\right)$. Further, using the topology induced from the measure with $\mathrm{e}^{-\beta \mathcal{E}(Z)}$, we could treat wider class of function as extension of spaces $\mathcal{C}^{\omega}\left(S^{1}, \mathbb{R}\right)$ and $\mathcal{C}^{\omega}\left(S^{1}, \mathbb{C}\right)$. 


\subsection{Isometry Deformation: Goldstein-Petrich Scheme}

In order to determine the equi-energy flow, we will also use the Goldstein-Petrich scheme $[24,46]$. For a deformation parameter $t$ and the arclength $s$, the non-stretch (isometry) deformation means

$$
\left[\partial_{t}, \partial_{s}\right] Z=0
$$

From Proposition 2, the non-stretch condition requires that the deformation must be expressed by

$$
\partial_{t} k=\Omega U_{i}, \quad k U_{i}=\partial_{s} U_{r}
$$

where

$$
\Omega:=\left(\partial_{s}^{2}+\partial_{s} k \partial_{s}^{-1} k\right)
$$

is a pseudo-differential operator which is known as the recursion operator of the MKdV hierarchy in the soliton theory [24].

On the other hand, since the deformation is treated as inner symmetry of the quantized elastica, the deformation should contain the trivial non-stretch deformation, which corresponds to freedom of choice of the origin of the arclength along the loop

$$
\partial_{t} \phi=-\partial_{s} \phi \equiv k, \quad \partial_{t} k=-\partial_{s} k .
$$

This is the trivial $\mathrm{U}(1)$ action on every loop space, which, of course, preserves the Euler-Bernoulli energy (4) of our quantized elastica.

Let us consider here the general case of non-stretching deformation which preserves the Euler-Bernoulli energy (4). In general, the equi-energy condition for a deformation means

$$
\partial_{t} \oint \mathrm{d} s k^{2}=2 \oint \mathrm{d} s k \partial_{t} k=0 .
$$

We have the following lemma and proposition:

Lemma $12([\mathbf{4 6 , 5 6 ] )}$ The non-stretching equi-energy deformation with respect to deformation parameter $t$

$$
\partial_{t} k=\Omega U_{i}=: f \in \mathcal{C}^{\omega}\left(S^{1}, \mathbb{R}\right)
$$

means that there exists a function $g \in \mathcal{C}^{\omega}\left(S^{1}, \mathbb{R}\right)$ such that

$$
k f=\partial_{s} g
$$


Proof: Every $h \in \mathcal{C}^{\infty}\left(S^{1}, \mathbb{R}\right)$ satisfying $\oint \mathrm{d} s h(s)=0$ is integrable, i.e., there exists $a \in \mathcal{C}^{\infty}\left(S^{1}, \mathbb{R}\right)$ such that $h=\partial_{s} a$. The above is confirmed by the relations

$$
\partial_{t} \oint k^{2} \mathrm{~d} s=2 \partial_{t} \oint k \partial_{t} k \mathrm{~d} s=2 \oint k f \mathrm{~d} s=\oint \partial_{s} g \mathrm{~d} s=0 .
$$

Proposition 13. The non-stretching equi-energy deformation $\partial_{t} Z$ induces another non-stretching deformation $\partial_{t^{\prime}} Z=(f+\sqrt{-1} g) \partial_{s} Z\left(U_{i}, f\right.$ and $g$ are as in Lemma 12)

$$
\partial_{t^{\prime}} k=\Omega f=\Omega \partial_{t} k=\Omega^{2} U_{i}
$$

Proof: This is obvious from Lemma 1 and (36).

\subsection{Quantized Elastica: MKdV Hierarchy}

Let us consider the non-stretching equi-energy deformation $\left(t_{1}, t_{2}, t_{3}, \ldots\right)$ which includes the trivial $\mathrm{U}(1)$ action and all of the induced deformations described in Proposition 13. It implies that we consider the deformation $\left(t_{1}, t_{2}, t_{3}, \ldots\right)$ given by

$$
\partial_{t_{\ell}} k=\Omega \partial_{t_{\ell-1}} k=\Omega^{\ell-1} \partial_{s} k, \quad \ell=1,2,3, \ldots
$$

where

$$
\partial_{t_{1}} k=-\partial_{s} k
$$

This is the modified KdV hierarchy

$$
\begin{aligned}
& \partial_{t_{1}} k+\partial_{s} k=0 \\
& \partial_{t_{2}} k+\frac{3}{2} k^{2} \partial_{s} k+\partial_{s}^{3} k=0 \\
& \partial_{t_{3}} k+10 k \partial_{s} k \partial_{s}^{2} k+\frac{5}{2} k^{2} \partial_{s}^{3} k+\frac{5}{2}\left(\partial_{s} k\right)^{3}+\frac{15}{8} k^{4} \partial_{s} k+\partial_{s}^{5} k=0
\end{aligned}
$$

Here we should note that one can find a deformation that satisfies

$$
\partial_{t_{m}} k=0, \quad m>g .
$$

In this case, (37) with (38) becomes essentially finite relations. We will call such a deformation with finite $g$ a finite-type deformation. 
Let us call the integrals of the relation (37) with (38) in $\mathcal{M}_{\text {elas }}^{\mathbb{C}}$ the flow of the $\mathrm{MKdV}$ hierarchy or the MKdV flow. Then due to the integrability, the following lemma is well-known

Lemma 14. The flow of the $M K d V$ hierarchy preserve the energy functional

$$
\mathcal{E}[Z]:=\oint k^{2} \mathrm{~d} s
$$

which is the Euler-Bernoulli energy (4).

As the equations of the MKdV hierarchy is an initial problem, the solutions space contains every loop in the plane. Thus we have the proposition

Proposition 15. The $\mathrm{MKdV}$ flow acts on $\mathcal{M}_{\text {elas }, E}^{\mathbb{C}}$ and $\mathcal{M}_{\text {elas }, E}^{\mathbb{C}}$ is decomposed into the orbits of the flow.

Remark 16. Here I will give a comment on the relation between quantized elastica and the Schwarz derivative. For a loop $Z$, the Euler-Bernoulli energy functional (4) has another expression

$$
\mathcal{E}[Z]=\oint \mathrm{d} s\{Z, s\}_{\mathrm{SD}}
$$

where $\{Z, s\}_{\mathrm{SD}}$ is the Schwarz derivative

$$
\{Z, s\}_{\mathrm{SD}}=\partial_{s}\left(\frac{\partial_{s}^{2} Z}{\partial_{s} Z}\right)-\frac{1}{2}\left(\frac{\partial_{s}^{2} Z}{\partial_{s} Z}\right)^{2}=-\sqrt{-1} \partial_{s} k-\frac{1}{2} k^{2}
$$

which is basically the same as $x$ in (22). It is known as the Miura transformation [39] and the Schwarz derivative $\{Z, s\}$ SD obeys the $\mathrm{KdV}$ hierarchy when $k$ is governed by the MKdV hierarchy.

As showed in [56], behind the quantized elastica problem we have a loop space $\Omega S^{2}$ of $S^{2}$, i.e., the moduli of loops in the two sphere $S^{2}$ or the complex projective space $\mathbb{P}^{1}$. As we consider the quantized elastica as a loop space in the regular function category, we encounter the $\mathrm{KdV}$ flow and the moduli space is governed by the solution space

$$
\left(-\partial_{s}^{2}-\frac{1}{2}\{Z, s\}_{\mathrm{SD}}\right) \psi=0
$$

which was studied by Poincaré (1854-1912). 
Because it is well-known that the solution of the MKdV hierarchy is given by the hyperelliptic curves including $\infty$ genus, it is worth to include the following remark.

Remark 17. As the Euler's elastica is related to the elliptic functions, the quantized elastica is related to the hyperelliptic functions, and naturally contains the Euler's elastica. From Proposition 15, we can classify the quantized elastica $\mathcal{M}_{\text {elas }}^{\mathbb{C}}$ using the solution space of the differential equations as in the classical elastica problem. It is natural to deal with the moduli space of the solution space of the MKdV hierarchy as Euler did for the classical elastica and the SMKdV equation though treatment of the moduli space of solution space of the MKdV hierarchy is very complicate.

Then we have our theorem:

Theorem $18([46,56]) \mathcal{M}_{\text {elas, } E}^{\mathbb{C}}$ is characterised by solution space of the MKdV hierarchy and hyperelliptic curves.

\subsection{Quantized Elastica: Solution Space of the MKdV Hierarchy}

In this subsection, we will show the construction of the finite-type deformation explicitly. For a natural number $g(g=1,2,3, \cdots)$ and a hyperelliptic curve $C_{g}$

$$
\begin{aligned}
y^{2} & =x^{2 g+1}+\lambda_{2 g} x^{2 g} \cdots+\lambda_{1} x+\lambda_{0} \\
& =\left(x-e_{1}\right)\left(x-e_{2}\right)\left(x-e_{3}\right) \cdots\left(x-e_{2 g+1}\right) .
\end{aligned}
$$

We have showed that the solutions of the MKdV hierarchy is given by the tangential angle [50]

$$
\phi(s)=\frac{1}{\sqrt{-1}} \sum_{i=1}^{g} \log \sqrt{-1}\left(x_{i}(s)-e_{1}\right)
$$

where $\left(\left(x_{1}, y_{1}\right), \cdots,\left(x_{g}, y_{g}\right)\right) \in \mathbf{S}^{g}\left(C_{g}\right)$ and

$$
s=u_{g}, \quad u_{j}=\sum_{i=1}^{g} \int_{\infty}^{\left(x_{i}, y_{i}\right)} \mathrm{d} u_{j}, \quad \mathrm{~d} u_{j}=\frac{x^{j-1} \mathrm{~d} x}{y}, \quad j=1, \cdots, g .
$$

Here $\mathrm{S}^{g}\left(C_{g}\right)$ is the symmetric product space of $C_{g}$. The reason why the sum over $g$ terms appear is due to the fact that the parameter $u$ of the curve $C_{g}$ is a solution of the differential equation

$$
\frac{\mathrm{d}^{g}}{\mathrm{~d} x^{g}}\left(2 y \frac{\mathrm{d} u}{\mathrm{~d} x}\right)=0
$$


Here we should remark also that (42), (43), (44) and (45) are interpreted as generalization of (15), (23), (17) and (16) respectively. The correspondence

$$
u:=\left(u_{1}, \cdots, u_{g}\right): \mathrm{S}^{g}\left(C_{g}\right) \longrightarrow \mathbb{C}^{g}
$$

is called the Abel map. Speaking more precisely the left hand side should be regarded as the abelian universal covering of the $\mathrm{S}^{g}\left(C_{g}\right)$ because the integral depends upon the contour and winding numbers of the loop. The integral (44) is known as the incomplete hyperelliptic integral of the first kind, whose complete version gives the period of the hyperelliptic functions

$$
\omega_{i, j}^{\prime}:=\int_{\infty}^{\left(e_{2 i-1}, 0\right)} \mathrm{d} u_{j}, \quad \omega_{i, j}^{\prime \prime}:=\int_{\infty}^{\left(e_{2 i}, 0\right)} \mathrm{d} u_{j} .
$$

Let $\Lambda$ denote the lattice in $\mathbb{C}^{g}$ generated by $\left(2 \omega^{\prime}, 2 \omega^{\prime \prime}\right)$. Using $\Lambda$, the Jacobi variety $\mathcal{J}_{g}$ is defined by

$$
\kappa: \mathbb{C}^{g} \rightarrow \mathcal{J}_{g}=\mathbb{C}^{g} / \Lambda
$$

$\mathcal{J}_{g}$ and $\mathrm{S}^{g}\left(C_{g}\right)$ are birational without depending upon the contours in the Abel map. The unnormalised differentials of the second kind are defined by

$$
\mathrm{d} r_{j}:=\frac{1}{2 y} \sum_{k=j}^{2 g-j}(k+1-j) \lambda_{k+1+j} x^{k} \mathrm{~d} x, \quad j=1, \cdots, g
$$

which lead also to the complete hyperelliptic integral, of the second kind

$$
\eta_{i, j}^{\prime}=\int_{\infty}^{\left(e_{2 i-1}, 0\right)} \mathrm{d} r_{j}, \quad \eta_{i, j}^{\prime \prime}:=\int_{\infty}^{\left(e_{2 i}, 0\right)} \mathrm{d} r_{j} .
$$

Then the Legendre relation in Proposition 6 is generalized to

$$
\eta^{\prime} \omega^{\prime \prime}-\omega^{\prime} \eta^{\prime \prime}=\frac{\pi}{2}
$$

Historically it is very interesting that this formula essentially appeared in a letter of Galois (1811-1832) to Chevalier 1832 [23].

Corresponding to Weierstrass sigma function (21), it is convenient to introduce the Klein's sigma function [36] (see the references [7, 12, 13]). Let $\mathbb{T}=\omega^{\prime-1} \omega^{\prime \prime}$. The theta function on $\mathbb{C}^{g}$ with modulus $\mathbb{T}$ and characteristics $\mathbb{T} a+b$ is given by

$$
\begin{aligned}
\theta\left[\begin{array}{l}
a \\
b
\end{array}\right](z) & =\theta\left[\begin{array}{l}
a \\
b
\end{array}\right](z ; \mathbb{T}) \\
& =\sum_{n \in \mathbb{Z}^{g}} \exp \left[2 \pi \mathrm{i}\left\{\frac{1}{2}^{t}(n+a) \mathbb{T}(n+a)+{ }^{t}(n+a)(z+b)\right\}\right]
\end{aligned}
$$


for $g$-dimensional complex vectors $a$ and $b$. The $\sigma$-function ( [3], p.336, [4, 5, 12, $13])$, an entire function on the space $\mathbb{C}^{g}$, is given by the formula

$$
\sigma(u)=\gamma_{0} \exp \left\{-\frac{1}{2}{ }^{t} u \eta^{\prime} \omega^{\prime-1} u\right\} \vartheta\left[\begin{array}{l}
\delta^{\prime \prime} \\
\delta^{\prime}
\end{array}\right]\left(\frac{1}{2} \omega^{\prime-1} u ; \mathbb{T}\right)
$$

where $\delta$ and $\delta^{\prime}$ are half-integer characteristics giving the vector of Riemann constants with basepoint at $\infty$ and $\gamma_{0}$ is a certain non-zero constant. The $\sigma$-function vanishes only on $\kappa^{-1}\left(W_{g-1}\right)$, where $W_{k}$ is the image of the Abelian map which is restricted over $S^{k}\left(C_{g}\right)$ (see for example [3], p.252). The Kleinian $\wp$ and $\zeta$ functions are defined by

$$
\wp_{i j}=-\frac{\partial^{2}}{\partial u_{i} \partial u_{j}} \log \sigma(u), \quad \zeta_{i}=\frac{\partial}{\partial u_{i}} \log \sigma(u)
$$

which correspond to (20).

Using these tools, the quantized elastica as a generalization of Theorem 7 is obtained.

Theorem $19\left(\left[\mathbf{5 0 , 5 1 ] )} Z \in \mathcal{M}_{\text {elas, } E}^{\mathbb{C}}\right.\right.$ is given by $s:=u_{g}+s_{0}$

1.

$$
\partial_{s} Z=\operatorname{al}_{r}\left(u+s_{0}\right)^{2}=\left(e_{r}-x_{1}\right)\left(e_{r}-x_{2}\right) \cdots\left(e_{r}-x_{g}\right)
$$

where $\mathrm{al}_{r}$ is the Weierstrass al-function defined by

$$
\operatorname{al}_{r}(u):=\sqrt{\left(e_{r}-x_{1}\right)\left(e_{r}-x_{2}\right) \cdots\left(e_{r}-x_{g}\right)}=\gamma_{0}^{\prime} \frac{\mathrm{e}^{-\eta_{r} u} \sigma\left(u+\omega_{r}\right)}{\sigma\left(\omega_{r}\right) \sigma(u)} .
$$

Here $\omega_{r}:=\int_{\infty}^{\left(e_{r}, 0\right)} \mathrm{d} u, \eta_{r}:=\int_{\infty}^{\left(e_{r}, 0\right)} \mathrm{d} r$ and $\gamma_{0}^{\prime}$ is a constant number.

2.

$$
Z=e_{r}^{g} s-\sum_{i=1}^{g} \zeta_{i} e_{r}^{i-1}
$$

3. Further $\mathcal{M}_{\text {elas }}^{\mathbb{C}}$ is decomposed to

$$
\mathcal{M}_{\text {elas }}^{\mathbb{C}}=\cup_{g=0}^{\infty} \mathcal{M}_{\text {elas }, g}^{\mathbb{C}}
$$

where $\mathcal{M}_{\text {elas, } g}^{\mathbb{C}}$ is a subspace of $\mathcal{M}_{\text {elas }}^{\mathbb{C}}$ whose elements are written by the hyperelliptic functions of genus $g$. 
Remark 20. The hyperelliptic Weierstrass al-function $\mathrm{al}_{r}$ should be interpreted as a generalization of Jacobians sn, dn, cn functions [79] and related to Mumford's base [59]. The above expressions of $\partial Z$ and $Z$ in terms of the polynomial of $x$ and the Kleinian zeta function should be also regarded as a generalization of Theorem 7. The above expression of $\mathrm{al}_{r}$ in terms of the Kleinian sigma functions is a generalization of (27).

$Z\left(u \in \mathcal{J}_{g}\right)$ specifies the equi-energy flows which we obtain. As in elastica, the reality condition $\left|\partial_{s} Z\right|=1$ and $s \in \mathbb{R}$ is very important even in quantized elastica. In [52], the reality condition is studied corresponding to Proposition 11.

Recently Calini and Ivey [15] showed more realistic shape of vortex filament in three dimensional space. The solution structure of vortex filament is closely related to the quantized elastica [18].

Remark 21. Regarding various problems, i.e., 1) determination of the equi-energy flow (orbit), 2) evaluation of the volume of the flow (orbit) $\operatorname{vol}\left(\mathcal{M}_{\text {elas, } E}^{\mathbb{C}}\right), 3$ ) determination of these distributions over $\{E\}=\mathbb{R}_{\geq 0}$, and sum them $\{E\}=\mathbb{R}_{\geq 0}$, we have shown that the equi-energy flow as MKdV hierarchy is obtained. It implies that (1) is partially settled but the closed condition $Z(s)=Z(s+\omega)$ for a certain $\omega \in \mathbb{R}$ is not yet. Further (2) the evaluation of the volume of the flow (3) determination of these distributions over $\{E\}=\mathbb{R}_{\geq 0}$, and sum them $\{E\}=\mathbb{R}_{\geq 0}$ remain. However it should be noted that the remaining problems are very difficult and, still, open. For example, the problem to evaluate the volume of the equi-energy flows is reduced to the problem to investigate of the real part of hyperelliptic Jacobians and their moduli.

In order to solve the open problem, let us mention a few quite different approaches.

\subsection{Frenet-Serret Relation and Dirac Operator: Fermionic Approach}

In this subsection, we will describe the submanifold Dirac operator. It turns out that the Frenet-Serret relation (2) can be regarded as one-dimensional submanifold Dirac equation $[53,54,57]$

$$
\not D:=\left(\begin{array}{cc}
\partial_{s} & -v \\
v & \partial_{s}
\end{array}\right), \quad \not D\left(\begin{array}{c}
\sqrt{\partial_{s} Z} \\
\sqrt{-1} \sqrt{\partial_{s} Z}
\end{array}\right)=0
$$

where $v=k / 2$. The Dirac operator is defined by the restriction to spinor over a loop of the Dirac operator in the spin bundle over the plane $\mathbb{C}$. The external field 
$v$ comes from the normalisation of the Hilbert space and thus is not trivial from the viewpoint $[53,54]$.

As mentioned above, behind the quantized elastica problem we have (41). The quadratic reciprocity law in the number theory which Euler partially studied gives the data of splitting law when we extend the integers $\mathbb{Z}$ to the Gauss integers $\mathbb{Z}[\sqrt{-1}]$. For example, in $\mathbb{Z}[\sqrt{-1}]$, the prime number 13 in $\mathbb{Z}$ is decomposed as $(3+2 \sqrt{-1})(3-2 \sqrt{-1})$. The Dirac operator appears when we extend the scalar field to the spinor field which has several components. Probably the relation between (41) and (47) could be interpreted via the theory of quadratic forms.

Here we will present only the list of the results from $[45,57]$.

Theorem $22([45,57])$ Assume that the curve $Z$ is given.

1. The partition function of the fermionic field over $Z$

$$
\mathcal{Z}_{f}[Z]=\int D \psi \exp \left(-\beta \int_{Z} \mathrm{~d} s \bar{\psi} \not D \psi\right)
$$

is reduced to

$$
\mathcal{Z}_{f}[Z]=\exp (-\beta \mathcal{E}[Z]) .
$$

2. The eigenvalue preserving deformation of $\not D$ or deformation of $Z$ is equal to that the half of the curvature obeys the MKdV hierarchy. It is known as the fact in the inverse scattering method.

3. The Atiyah-Singer index of $\not D$ is the winding number of the loop

$$
\text { index } \not D=\int \mathrm{d} s \partial_{s} \varphi \text {. }
$$

The fermionic approach basically gives us every information, including the topological data and even analytic data. The solution of $\not D \psi=0$ is given by alfunction which is a ratio of $\sigma$-functions. Thus the analytic properties of the Dirac operator is also directly connected with the Kleinian sigma functions and Weierstrass al functions.

Some of these properties are generalized to those over every spin-submanifold immersed in a spin-manifold $[53,54]$. The anomaly or the local index theorem for the Dirac operator over a conformal surface immersed in $\mathbb{R}^{3}$ gives the action integral including that of the extrinsic Polyakov string [63] and the Willmore energy [49]. Then the quantized elastica is generalized to the quantization of the Willmore energy [47]. These objects were also investigated well by Arroyo, Barros and Garay [1, 25], Kholodenko and Nesterenko [33], Konopelchenko [38], Carroll [9] and Taimanov [69] and many others. 


\subsection{Loop Space Cohomology}

In this subsection, we will comment another topological data. The quantized elastica problem requires that we characterise the singularities among elasticas related to curves with different genus. To do so, we have to deal with the moduli of the real hyperelliptic curves.

As a first step, we will consider the cohomological properties of the moduli space $\mathcal{M}_{\text {elas }}^{\mathbb{C}}$ or the loop space in the category of regular functions $[8,11,56]$. Thus it is naturally to mention that a cohomology ring of the moduli space $\mathcal{M}_{\text {elas }}^{\mathbb{C}}$ is a ring isomorphic to that of loop space $\Omega S^{2}$ of $S^{2}[8,56]$

$$
\mathcal{H}^{q}\left(\mathcal{M}_{\text {elas }}^{\mathbb{C}}, \mathbb{R}\right)=\mathcal{H}^{q}\left(\Omega S^{2}, \mathbb{R}\right)=\mathbb{R} \quad \text { for } \quad q \geq 0 .
$$

It is very interesting that the generator of the cohomology ring over $\mathcal{M}_{\text {elas }}^{\mathbb{C}}$ corresponds to the operator which jump from the genus $g$ to $g+1$ of the moduli of the hyperelliptic curves [56]. Further (48) can be regarded as a categorical relation because there is a natural forgetful functor from $\mathcal{M}_{\text {elas }}^{\mathbb{C}}$ to $\Omega S^{2}$.

If the quantized elastica problem is generalized to one over $\mathbb{R}^{3}$ [48], the relation (48) can be also obtained over $\Omega S^{3}$. Besides the fermionic approach suggests the way for the generalization to higher dimensional quantized elastica [43] via the generalized MKdV equation, and it might be not difficult to have more generalized relations.

\subsection{Baby Quantized Elastica}

Finally we should give a toy model on the partition function (35). In the partition function (35), the winding effects should be also considered. When the curves intersect at a point with infinite intersection degree in the analytic function category, it means that the curves agree completely, and in the moduli space $\mathcal{M}_{\text {elas }}^{\mathbb{C}}$, they are multi-winding of a loop.

We can not compute the partition function itself in this stage but we can handle its part, $\mathcal{Z}^{(g)}[\beta], g=0,1$ which consists only of the closed loop of each $g=0$ and $g=1$ with winding number effect. We know the closed loop solutions of genus zero and one explicitly, which is given by disjoint types, i.e., a circle and an figure-eight shape. Considering contributions of winding of the loop for each $g=0$ and $g=1$, we obtain

$$
\mathcal{Z}^{(g)}[\beta]=\sum_{n=1}^{\infty} \mathrm{e}^{-\beta n^{2} E_{g}}=\frac{1}{2}\left(\theta\left(\sqrt{-1} \beta E_{g} / \pi\right)-1\right)
$$


where $E_{0}$ and $E_{1}$ are the Euler-Bernoulli energies (39) of genera zero and one and $\theta(z)$ is the elliptic theta function, $\theta(z):=\sum_{n=-\infty}^{\infty} \mathrm{e}^{\sqrt{-1} \pi z n^{2}}$. Due to properties of the elliptic theta function and Poisson sum formula

$$
\mathcal{Z}^{(g)}[\beta]=\sqrt{\frac{1}{E_{g} \beta}} \sum_{n=1}^{\infty} \mathrm{e}^{-n^{2} / E_{g} \beta}+\frac{1}{2}\left(\frac{1}{\sqrt{E_{g} \beta}}-1\right) .
$$

As $\mathcal{Z}^{(g)}\left[\beta+2 \pi \sqrt{-1} / E_{g}\right]=\mathcal{Z}^{(g)}[\beta]$, it is clear that such $\mathcal{Z}^{(g)}[\beta]$ has modular properties.

When it is possible to approximate $\mathcal{Z}[\beta]$ by $\mathcal{Z}^{(g)}[\beta]$ or $\mathcal{Z}^{(0,1)}[\beta]:=\sum_{g=0}^{1} \mathcal{Z}^{(g)}[\beta]$ in a certain sense and consider a perturbation of this model, we might encounter a critical phenomena from the viewpoint of statistical physics, due to the modular properties.

\subsection{Final Remark on Quantized Elastica}

The elastica problem rested on the great progress of mathematics and physics in the eighteenth century and the quantized elastica problem also needs adequate progress as well. Its goal is to evaluate the partition function and its related expectation values.

However we should emphasize that the quantized elastica belongs to the category of the real analytic geometry, rather than categories of complex analytic geometry and topology. In a scattering problem of quantum physics, the asymptotic behaviour can be expressed in terms of trigonometric functions. Thus soliton solutions, which are given by trigonometric functions, more precisely hyperbolic functions, and punctured Riemann surfaces play crucial roles there. The topological properties might determine the problem completely. However in the quantized elastica, its topology is stronger than that of the scattering problems.

Of course, it is not an object in the category of topology though, of course, the forgetful functor works like (48). However non-stretching condition is a stiff condition and does not enable us to deal with perturbative approach. Topological approach is necessary but it is not sufficient in the quantized elastica problem.

Further the reality condition of the quantized elastica requires the real analytic treatment rather than complex one. Further, whereas the string theory is connected with Riemann surfaces by regarding them as the strings themselves, quantized elastica is expressed by hyperelliptic zeta functions with the reality condition. In other words, whereas the string theory is purely algebro-geometrical over $\mathbb{C}$, the quantized elastica is a transcendental object over $\mathbb{R}$. 
For approaching the goal, we need a deeper knowledge of the moduli of real hyperelliptic curves, the behaviour of the hyperelliptic zeta functions, properties of meromorphic functions related to the hyperelliptic Jacobian, differential geometrical properties of loop space and so on. In the above theories, one makes use of the theory of hyperelliptic function theory and its relation to integrable systems which are based upon Baker's works [3-5], Mumford's theory in [59], and works of Russian-Ukrainian groups - Belokolos, Bobenko, Enol'skii, Its, and Matveev [7] and Buchstaber, Enolskii and Leykin [12,13]. For example, as Enolskii have pointed out, the unpublished corrected version of [12] gave also a relation which leads to more algebraic expression of the above results of Theorem 19 here or Theorem 2 in [50]. For the goal, we need the development of the novel hyperelliptic function theory based upon [12,13]. Especially in [14], Buchstaber and Leykin have constructed a new theory of sigma functions including one related to the hyperelliptic curves, which, hopefully will help us to solve the problem.

It is expected also that the fermionic approach, the cohomological approach, and the results for the baby quantized elastica might give another way to the goal.

\section{Discussion}

The mathematics might be regarded as a language in the meaning of Barthes [6]. If it was a kind of a language or the ecriture, it might have dialects and some constraints on the places where it could be used or not [6].

Using modern terminology, the works of Bernoulli's and Euler on the elastica problem are closely related to the harmonic map theory, differential geometry, algebraic geometry, moduli of elliptic functions, loop space and so on. If one who knows modern mathematics restricts himself with a single language (field) with a dialog and limitations, he could not deeply understand the problem as Euler did. In fact, the elastica problem came from the studies of strings and beams, which have thickness, various cross sections and other individual characteristics. It is difficult to give the definition from the first, following style in the modern pure mathematics. Indeed, in the real scientific and physical problems, there are so many undefined concepts generally, especially in the problems of the shape.

James Bernoulli introduces the elastica problem by extracting the essentials of the above mentioned problems. However it is not within a single category as Bernoulli's and Euler studied it without persisting on such opinion and finally created the theory of elastica. Their style of study is that they extracted the essen- 
tials, purified the discovered facts and tools as pure science, and using them, they discovered surprising facts and solve the problem. Elastica problem is a prototype of such approach.

In the beginning of the twentieth century, Klein (1849-1925) wrote "Diese Tendenz, die Wissenschaft nicht nur in immer zahlreichere Einzelkapitel zu zerlegen, sondern Schulunterschiede nach der Art der Behandlung zu schaffen, würde, wenn sie einseitig zur Geltung käme, den Tod der Wissenschaft herbeiführen" ${ }^{1}$ [35, p.327]. In spite of Klein's concern, the studies in the twentieth century had developed by breaking to pieces.

When we look over the studies of Bernoulli's and Euler from a viewpoint of mathematical science, we might feel their spirits, "When one expresses a (physical) object in terms of mathematics, he must search the most proper language to represent it. If the language does not exist, he should construct a new language to describe it without hesitating." Even though only few persons can do it like Euler and Bernoulli's, we should inherit their spirit. Studying mathematics transversally might enable us to revive the studies which died as in the statement of Klein. At least, we hope that here we have showed that in order to solve the quantized elastica problem, we need such studies.

\section{Acknowledgements}

This work is dedicated to my grandfather's uncle and foster father, Chūhachi Ninomiya (1866-1936), who studied aeroplane alone and invented a powered aeroplane before the first flight of Wright brother's Flyer $1 .^{2}$ Even after his distinct study was denied by the Japan authorities in those days, he continued the study alone working as a businessman and pharmacist in a company until he knew the first flight. The present author is also a manager, engineer, and applied scientist in a company in the daytime. One of the reasons for studying mathematics and pure science for two decades is due to the existence of Chühachi. The difference from him is that there are so many friends, colleagues, teachers in the world who encourage and support my work, which is the other reason.

I would like to express my sincere thanks to Kenichi Tamano, Satoru Saito, Hideo

\footnotetext{
${ }^{1}$ This could be translated into English as follows:"This tendency, to decompose the academy of science not only into ever more numerous separate chapters but also to create schools differences according to such treatment, would bring about the death of the science if it became effective on one side."

${ }^{2}$ http://en.wikipedia.org/wiki/Chūhachi_Ninomiya
} 
Tsuru, Tetsuji Tokihiro, Kiyoshi Sogo, Shin Takagi, Hiroshi Kuratsuji, Norio Konno, Hideo Mitsuhashi, Arkady Kholodenko, Shinsuke Watanabe, Junkichi Satsuma, Kohtaro Watanabe, Katsuhiro Nishinari, Shingo Ishiwata, and to many professors and peoples in the related universities, for their support. Due to them, I could go on to study the integrable systems. This study started when Hideo Tsuru pointed out to me the relation between elastica and DNA. The study of hyperelliptic function is due to discussions with Yoshihiro Ônishi, Victor Enoliskii, Chris Eilbeck, and Emma Previato. I would like to mention also that I have learned many things in mathematics, from the basic to the advanced one, from Kenichi Tamano for two decades. I am also grateful to many colleagues over the world - John McKay, Emma Previato, Yuji Kodama, Martin Guest, Franz Pedit, Boris Konopelchenko, Iskander Taimanov, Robert Carroll, Yoshihiro Ohnita, Junichi Yamashita, Mikio Nakahara, Yasuhide Fukumoto, Satoshi Kawakubo and Ivaïlo Mladenov for their continious interest in this problem.

\section{References}

[1] Arroyo J., Barros M. and Garay O., Willmore-Chern Tubes on Tomogeneous Spaces in Warped Product Spaces, Pacific. J. Math. 188 (1999) 201-207.

[2] Bell E., Men of Mathematics, Penguin Books Ltd., New York, 1937, (translated to Japanese by Y. Tanaka and H. Ginbayashi, Tokyo-tosho, Tokyo 1976).

[3] Baker H., Abelian Functions - Abel's Theorem and the Allied Theory Including the Theory of the Theta Functions, Cambridge Univ. Press, Cambridge, 1897, reprinted in 1995.

[4] Baker H., On the Hyperelliptic Sigma Functions, Am. J. Math. 20 (1898) 301-384.

[5] Baker H., On a System of Differential Equations Leading to Periodic Functions, Acta Math. 27 (1903) 135-156.

[6] Barthes R., Le degre zero de l'ecriture, French \& European Pubns, Paris, 1953.

[7] Belokolos E., Bobenko A., Enol'skii V., Its A. and Matveev V., AlgebroGeometric Approach to Nonlinear Integrable Equations, Springer, Berlin, 1994.

[8] Bott R. and Tu L., Differential Forms in Algebraic Topology, Springer, Berlin, 1986. 
[9] Carroll R., Quantum Theory, Deformation and Integrablility, NorthHolland, Amsterdam, 2000.

[10] Bryant R. and Griffiths P., Reduction for Constrained Variational Problems and $\int \frac{\kappa^{2}}{2} \mathrm{~d} s$, Amer. J. Math. 108 (1986) 525-570.

[11] Brylinski J-L., Loop Spaces, Characteristic Classes and Geometric Quantization, Birkhäuser, Boston, 1992.

[12] Buchstaber V., Enolskii V. and Leykin D., Kleinian Functions, Hyperelliptic Jacobians and Applications, Reviews in Mathematics and Mathematical Physics (London), S. Novikov and I. Krichever (Eds), Gordon and Breach, India, 1997, pp 1-125.

[13] Buchstaber V., Enolskii V. and Leykin D., Hyperelliptic Kleinian Functions and Application, Amer. Math. Soc. Transl. 179 (1997) 1-33.

[14] Buchstaber V. and Leykin D., Addition Laws on Jacobian Varieties on Plane Algebraic Curves, Proc. Steklov Inst. Math. 251 (2005) 1-72.

[15] Calini A. and Ivey T., Finite-gap Solutions of the Vortex Filament Equation: Isoperiodic Deformations, J. Nonlinear Sci. 17 (2007) 527-567.

[16] Chirikjian G., The Stochastic Elastica and Excluded-Volume Perturbations of DNA Conformational Ensembles, Int. J. Non-Linear Mech. 43 (2008) 1108-1120.

[17] Djondjorov P., Hadzhilazova M., Mladenov I. and Vassilev V., Explicit Parameterization of Euler's Elastica, In: Proceedings of the Ninth International Conference on Geometry, Integrability and Quantization, I. Mladenov (Ed), SOFTEX, Sofia 2008, pp 175-186.

[18] Eilbeck J., Enolskii V. and Kostov N., Quasiperiodic and Periodic Solutions for Vector Nonlinear Schrödinger Equations, J. Math. Phys. 41 (2000) 8236-8254.

[19] Euler L., Methodus Inveniendi Lineas Curvas Maximi Minimive Proprietate Gaudentes, 1744, Leonhardi Euleri Opera Omnia Ser. I vol. 14.

[20] Fellmann E., Leonhard Euler, Rowohlt Taschenbuch, 1995, (translated into Japanese by Yamamoto A., Spinger-Tokyo, Tokyo 2002).

[21] Fukumoto Y., Analogy Between a Vortex-jet Filament and the Kirchhoff Elastic Rod, Fluid Dyn. Res. 39 (2007) 511-520.

[22] Galilei G., Discorisi e Dimostrazioni Matematiche Intorne a due Nuove Scienze, Elfevirii, 1638, (translated into Japanese by T. Konno and S. Hida, Iwanami, Tokyo 1937). 
[23] Galois E., Euvres Mathématiques d'Évariste Galois, J. de math. pures et appliquées 11 (1846) 381-444, (tranlated into Japanese by Takase M, Abel/Galois Elliptic Functions. Asakura-shoten, Tokyo, 1998).

[24] Goldstein R. and Petrich D., The Korteweg-de Vries Hierarchy as Dynamics of Closed Curves in the Plane, Phys. Rev. Lett. 67 (1991) 3203-3206.

[25] Garay O., Extremals of the Generalized Euler-Bernoulli Energy and Applications, J. Geom. Symm. Phys. 12 (2008) 27-61.

[26] Goss V., The History of the Planar Elastica: Insights into Mechanics and Scientific Method, Sci. \& Educ. 18 (2009) 1057-1082.

[27] Hasimoto H., A Soliton on a Vortex Filament, J. Fluid Mech. 51 (1972) 477-485.

[28] Houzel C., Abrég'e d'histoire des mathématiques, vols. 1\&2, J. Dieudonné (Ed), Hermann, Paris, 1978 (translated into Japanese by K. Ueno, N. Kaneko, Y. Namikawa, Y. Morita and J. Yamashita, Iwanami, Tokyo 1985).

[29] Hurwitz A., Üeber die entwicklungscoefficienten der lemniscratischen functionen, Math. Ann. 51 (1899) 196-226.

[30] Isimori Y., On the Modified Korteweg-de Vries Soliton Equation and the Loop Soliton, J. Phys. Soc. Japan 50 (1981) 2471-2472.

[31] Kawakubo S., Kirchhoff Elastic Rods in Three-Dimensional Space Forms, J. Math. Soc. Japan 60 (2008) 551-582.

[32] Kholodenko A., Statistical Mechanics of Semiflexible Polymers: Yesterday, Today and Tomorrow, J. Chem. Soc. Faraday Trans. 91 (1995) 2473-2482.

[33] Kholodenko A. and Nesterenko V., Classical Dynamics of the Rigid String from the Willmore Functional, J. Geom. Phys. 16 (1995) 15-26.

[34] Kirchhoff G., Über das gleichgewicht und die bewegung einer elastischen scheibe, J. für reine und angew. math. 40 (1850) 51-88.

[35] Klein F., Vorlesungen über die Entwicklung der Mathematik im 19 Jahrhundert I, Springer, Berlin, 1926.

[36] Klein F., Über hyperelliptische sigmafunctionen, Math. Ann. 32 (1888) 351-380.

[37] Konno K., Ichikawa Y. and Wadati M., A Loop Soliton Propagating Along a Streched Rope, J. Phys. Soc. Jpn. 50 (1981) 1025-1026.

[38] Konopelchenko B., Weierstrass Representations for Surfaces in 4D Spaces and Thier Integrable Dynamics via the DS Hierarchy, Ann. Global Anal. Geom. 18 (2000) 61-74. 
[39] Lamb G., Elements of Soliton Theory, John Wiley \& Sons, New York, 1980.

[40] Langer J. and Singer D., The Total Squared Curvature of Closed Curves, J. Diff. Geom. 20 (1984) 1-22.

[41] Levien R., The Elastica: A Mathematical History, EECS Department, University of California, Berkeley, UCB/EECS-2008-103 2008 http://www.eecs.berkeley.edu/Pubs/TechRpts/2008/EECS-2008-103.html.

[42] Lyubchenko Y., Shlyakhtenko L., Aki T. and Adhya S., Atomic Force Microscopic Demonstration of DNA Looping GalR and HU, Nucl. Acids Research 25 (1997) 873-876.

[43] Matsutani S., On the Physical Relation Between the Dirac Equation and the Generalized $m K d V$ Equation on a Thin Elastic Rod, Phys. Lett. A 189 (1994) 27-31.

[44] Matsutani S., The Relation of Lemniscate and a Loop Soliton as 3/2 and 1 Spin Fields along the Modified Korteweg-de Vries Equation, Mod. Phys. Lett. A 10 (1995) 717-721.

[45] Matsutani S., The Physical Realization of the Jimbo-Miwa Theory of the Modified Korteweg-de Vries Equation on a Thin Elastic Rod: Fermionic Theory, Int. J. Mod. Phys. A 10 (1995) 3091-3107.

[46] Matsutani S., Statistical Mechanics of Elastica on Plane: Origin of MKdV Hierarchy, J. Phys. A Math. \& Gen. 31 (1998) 2705-2725.

[47] Matsutani S., On Density of State of Quantized Willmore Surface: A Way to a Quantized Extrinsic String in $\mathbb{R}^{3}$, J. Phys. A: Math. \& Gen. 31 (1998) 3595-3606.

[48] Matsutani S., Statistical Mechanics of Non-stretching Elastica in Three Dimensional Space, J. Geom. Phys. 29 (1999) 243-259.

[49] Matsutani S., Immersion Anomaly of Dirac Operator on Surface in $\mathbb{R}^{3}$, Rev. Math. Phys. 11 (1999) 171-186.

[50] Matsutani S., Hyperelliptic Loop Solitons with Genus g: Investigation of a Quantized Elastica, J. Geom. Phys. 43 (2002) 146-162.

[51] Matsutani S., On a Relation of Weierstrass al-Functions, Int. J. Appl. Math. 11 (2002) 295-307.

[52] Matsutani S., Reality Conditions of Loop Solitons Genus g: Hyperelliptic am Functions, Electron. J. Diff. Eqns. 2007 (2007) 1-12.

[53] Matsutani, S., Generalized Weierstrass Relations and Frobenius Reciprocity, Math. Phys. Anal. Geom. 9 (2007) 353-369. 
[54] Matsutani S., Generalized Weierstrass Relation for a Submanifold $S^{k}$ in $\mathbb{E}^{n}$ Coming From Submanifold Dirac Operator, Advanced Studies in Pure Mathematics 51 (2008) 259-283.

[55] Matsutani S., Relations in a Quantized Elastica, J. Phys. A: Math.\& Theor. 41 (2008) 075201 (12pp).

[56] Matsutani S. and Ônishi Y., On the Moduli of a Quantized Elastica in $\mathbb{P}$ and KdV Flows: Study of Hyperelliptic Curves as an Extension of Euler's Perspective of Elastica I, Rev. Math. Phys. 15 (2003) 559-628.

[57] Matsutani S. and Tsuru H., Physical Relation between Quantum Mechanics and Soliton on a Thin Elastic Rod, Phys. Rev. A 46 (1992) 1144-1147.

[58] Michor P. and Mumford D., Riemannian Geometries on Spaces of Plane Curves., J. Eur. Math. Soc. 8 (2006) 1-48.

[59] Mumford D., Tata Lecture on Theta II, Birkhauser, Boston, 1984.

[60] Mumford D., Elastica and Computer Vision, In: Algebraic Geometry and its Applications, C. Bajaj (Ed), Springer, Berlin, 1993 pp 507-518.

[61] Nishinari K., Nonlinear Dynamics of Solitary Waves in an Extensible Rod, Proc. R. Soc. Lond. A 453 (1997) 817-833.

[62] Pinkall U., Hopf Tori in $S^{3}$, Invent. Math. 81 (1985) 379-386.

[63] Polyakov A., Gauge Fields and Strings, Harwood, London, 1987.

[64] Prasolov V. and Solovyev Y., Elliptic Functions and Elliptic Integrals, Amer. Math. Soc., New York, 1993.

[65] Previato E., Geometry of the Modified KdV Equation, In: Geometric and Quantum Aspects of Integrable System, Lecture Notes in Physics vol. 424 pp.43-65, Springer, Berlin, 1993.

[66] Sachkov Y., Maxwell Strata in the Euler Elastic Problem, J. Dynamical and Control Systems 14 (2008) 169-234.

[67] Singer D., Lectures on Elastic Curves and Rods, In: Curvature and Variational Modeling in Physics and Biophysics, AIP Conference Proceedings 1002 (2008) pp 3-32.

[68] Sogo K., Variational Discretization of Euler's Elastica Problem, J. Phys. Soc. Japan 75 (2006) 064007.

[69] Taimanov I., Two-Dimensional Dirac Operator and the Theory of Surfaces, Russian Math. Suveys 61 (2006) 79-159.

[70] Toda M., Introduction to Elliptic Functions (in Japanese), Nihon-hyouronsha, Tokyo, 1976. 
[71] Truesdell C., The Rational Mechanics of Flexible or Elastic Bodies 16381788, Leonhardi Euleri Opera Omnia Ser. II vol. 11 Part 2, Orell Füssli, Zürich, 1960.

[72] Truesdell C., Essays in the History of Mechanics, Springer, New York, 1968.

[73] Truesdell C., History of Classical Mechanics Part II the 19th and 20th Centuries, Die Naturwissenschaften 63 (1976) 119-130.

[74] Truesdell C., The Influence of Elasticity on Analysis: The Classic Heritage, Bull. Amer. Math. Soc. 9 (1983) 293-310.

[75] Tsuru H., Equilibrium Shapes and Vibrations of Thin Elastic Rod, J. Phys. Soc. Japan 56 (1987) 2309-2324.

[76] Tsuru H. and Wadati M., Elastic Model of Highly Supercoiled DNA, Biopolymers 25 (1986) 2083-2096.

[77] Vassilev V., Djondjorov P. and Mladenov I., Cylindrical Equilibrium Shapes of Fluid Membranes, J. Phys. A 41 (2008) 435201(16pp).

[78] Watanabe K, Plane Domains which are Spectrally Determined, Ann. Global. Anal. Geom. 18 (2000) 447-475.

[79] Weierstrass K., Zur theorie der Abel'schen functionen, Aus dem Crelle'schen Journal 47 (1854) in Mathematische Werke I, Mayer und Müller, Berlin, 1894.

[80] Weil A., Number Theory, An Approach Through History From Hammurapi To Legendre, Birkhauser, Boston, 1983.

[81] Whittaker E. and Watson G., A Course of Modern Analysis, 4th ed. Cambridge Univ. Press, Cambridge, 1927.

[82] Willmore T., Riemannian Geometry, Oxford Univ. Press, Oxford, 1993.

Shigeki Matsutani

8-21-1 Higashi-Linkan Sagamihara

228-0811, Japan

E-mail address: rxb01142@nifty. com 